\title{
Electron correlations. II. Ground-state results at low and metallic densities
}

\author{
R. F. Bishop and K. H. Lührmann \\ Theoretical Physics Group, Department of Mathematics, University of Manchester Institute \\ of Science and Technology, P. O. Box 88, Manchester M6O 1QD, United Kingdom
}

(Received 19 May 1981; revised manuscript received 30 March 1982)

\begin{abstract}
In this second work in a series of papers the coupled-cluster or $\exp (S)$ formalism is applied to the problem of ground-state correlations in the one-component Fermi plasma. The main approximation employed is the so-called SUB2 approximation for the two-body subsystem correlation operator $S_{2}$ which provides a measure of the two-particle-two-hole component in the true ground-state wave function, and in which the coupling to larger subsystems is neglected. The SUB2 equations for Fermi systems are brought into tractable form by a "state-averaging" procedure which we develop by analogy with the comparable Bose equations, and which is shown by comparison with earlier exact results to be accurate in the metallic density regime at about the $1 \%$ level. Our final results for metallic densities include the completely integrated and self-consistent effects of the terms which by themselves generate (i) the random-phase approximation (RPA) and its associated exact long-range screening effects, (ii) the extra random-phase approximation exchange terms necessary to keep the RPA explicitly antisymmetric, (iii) the self-consistent particle-particle ladders (LAD) that describe two-particle scattering within the many-body medium and which describe the exact short-range behavior, (iv) a class of particle-hole ladder terms, and (v) the self-consistent hole-potential terms. Particular attention is paid to the important effects caused by the interference at intermediate separations of the long-range RPA and the short-range LAD effects. By comparison with recent and essentially exact stochastic simulations of the many-body Schrödinger equation, our results are seen to be accurate to about $1 \%$ for metallic densities, and hence to provide what is probably the currently best microscopic description available for this system. We show also that even in the low-density limit the SUB2 approximation provides a good "translationally-invariant-solid" description of both charged Fermi and Bose systems in this exact Wigner crystal regime.
\end{abstract}

\section{INTRODUCTION}

A general discussion of the coupled-cluster formalism of quantum many-body theory and its application to the properties of the ground state of the electron gas (otherwise known as the onecomponent Fermi plasma or jellium) has already been given in a previous paper ${ }^{1}$ by the present authors, hereafter referred to as I. In that paper attention was focused on the high-density limit, and the problem was solved in both the well-known random-phase approximation (RPA) and the not so well-known Tamm-Dancoff approximation (TDA). The solution of the RPA equations in the coupledcluster formalism was very considerably more complicated than in such alternate formalisms as the Green's-function formulation of timedependent perturbation theory, although the solution also contained much more information about the system. In the present paper one of our aims is to show that this initial disadvantage of the coupled-cluster formalism is much more than compensated for by the fact that the formalism seems particularly suited for the electron gas problem as soon as we go beyond RPA, and particularly if we wish to obtain a realistic description at metallic densities. In this second paper we therefore intend to extend the results of I for the ground-state properties of jellium over the remaining low- and metallic-density regimes, and we leave it to a third paper $^{2}$ to set up the formalism for excited states and to apply it to jellium.

Since we have no wish to repeat the introductory remarks nor the discussion of the coupled-cluster formalism already given in I, we content ourselves here with a brief reminder of the physical content of the formalism. The linked-cluster theorem for the exact many-body ground-state wave function 
[i.e., the usual $\exp (S)$ ansatz] is employed to decompose the $N$-body system into a set of mutually interacting $n$-body subsystems $(n \leq N)$ that are described by a set of so-called correlation operators $S_{n}$, whose matrix elements thus describe the excitation of $n$ particle-hole pairs from some suitably defined noninteracting $N$-body reference or model state. The exact $N$-body Schrödinger equation for the ground state is thereby exactly decomposed into a coupled set of $N$ (nonlinear) microscopic equations for the matrix elements of the correlation operators $S_{n}$. The general structure of this set of equations is that the $n$th equation in the hierarchy for $S_{n}$ is coupled, in the general case of the elementary particles comprising the system interacting via up to $j$-body forces, to the higher amplitudes $S_{n+i}$, with $1 \leq i \leq j$, as well as to all of the lower amplitudes $S_{m}$ with $m<n$. In our case, of course, we are interested only in $j=2$ Coulomb forces. In order for this exact reformulation of the many-body ground-state to be useful, this hierarchy of coupled equations has to be truncated using a suitable physically motivated approximation. The truncation scheme employed in I, and which we shall mostly, but not wholly, also employ here is the so-called SUB $n$ scheme in which all $S_{i}$ for $i>n$ are set equal to zero. The SUB1 approximation is just the familiar Hartree-Fock approximation, which itself is trivial for translationallyinvariant systems since in this case $S_{1} \equiv 0$ by momentum conservation. Thus the scheme with which we shall mostly concern ourselves is the SUB2 approximation which was discussed in great detail in I. For purposes of preliminary orientation it is important only to realize that the fermion SUB2 approximation contains as drastic subapproximations to itself such other familiar approximations as: (i) the RPA which, in the language of diagrammatic Goldstone perturbation theory, includes all the so-called ring or bubble diagrams, (ii) the analogous TDA in which only the "forwardgoing" (in time) bubble diagrams are retained, (iii) the approximation of the Bethe-Goldstone equation which sums the so-called particle-particle ladder (LAD) diagrams that describe the scattering of two particles inside the many-body medium, and (iv) the analogous Galitskii approximation which sums all particle-particle $(p p)$, hole-hole $(h h)$, and mixed $p p-h h$ ladders. We note that the SUB2 approximation also contains terms which allow to include in the two-body propagators that describe the intermediate scatterings in cases (iii) and (iv), for example, the (self-consistent) hole- and particle-potential terms that "renormalize" the bare (kinetic) "energy denominators" of these propagators. Thus, also (v) the whole of the usual Brueckner-Bethe-Goldstone theory applied so successfully to nuclear matter and to finite nuclei, is also embedded in the SUB2 approximation. In fact the SUB2 approximation is richer even than the fully self-consistent union of all the above approximations, but for full details we must refer the reader back to Sec. II of I. We now state that our main present aim is to apply to the electron gas, particularly in the interesting intermediate-coupling regime of metallic densities, the full SUB2 approximation (and even somewhat beyond); and hence to apply to this most well studied of all quantum many-body problems what is undoubtedly the most ambitious microscopic description it has yet received.

We stress again that the basic SUB2 approximation is exact apart from neglecting the interactions with higher-order subsystems. Otherwise, all twobody effects are included, and it is not surprising therefore that the SUB2 equations are complex. They have the structure for homogeneous, isotropic systems of a nonlinear integral equation for the matrix elements of the two-body correlation operator $S_{2}$, which are functions of three three-vectors. In order to gain some insight into these fermion equations, and to enable us to make physically sensible approximations to obtain their solutions, we are first motivated to look at the formally much simpler analogous Bose equations.

Hence in Sec. II the extension to bosons of the coupled-cluster formalism is outlined and we stress in particular the most interesting results obtained in the formally most difficult strong-coupling or low-density regime. For the Bose one-component plasma we find that at low densities the asymptotic analytic form of the ground-state energy is that appropriate for a solid rather than a fluid, even though our formalism from the outset assumes that both the reference or model state and the exact ground state are explicitly translationally invariant. We argue that the fact that we obtain such an ("amorphous") translationally-invariant description of the exact low-density Wigner crystal—or what one might wryly but aptly refer to as "set jellium"-is strongly indicative of the power of the coupled-cluster formalism and its SUB $n$ hierarchy of approximations. Thus even though the SUB2 description is constrained not to be able to describe properly the symmetry breaking that occurs at the physical phase transition between the high-density fluid and the low-density 
crystal phases, the formalism still seems able to describe the essential physics of the low-density regime. Thus what is in essence still a two-body subsystem approach, seems well able to describe qualitatively at least a phase that is an archetype of an $N$-body system where the $N$-body correlations dominate. We regard this result as one of several very strong pieces of evidence in favor of the SUB2 approximation being applicable even in situations that provide the most stringent tests for it. Finally, in Sec. II we deal also with the Fermi one-component plasma in the low-density limit on the basis of an approximation to the full SUB2 scheme that we develop later; and similar behavior is seen also in this case.

Thus motivated by the comparisons between Bose and Fermi systems, we develop in Sec. III our first approximations to the Fermi SUB2 scheme by suitably "state averaging" over the occupied states inside the Fermi sea, and we apply them first to the cases where we keep only the RPA terms and RPA exchange (RPAEX) terms necessary to keep this subapproximation explicitly antisymmetric. In the case of pure RPA we can check the validity of our approximation against the exact (analytical) results found in I; and a similar check is also made against the results of an exact (numerical) evaluation which goes beyond RPA by keeping the RPAEX terms in first order only. In both cases we find in the metallic-density regime agreement at the $1 \%$ level, and we argue that a similar level of agreement is to be expected in other cases where a direct check cannot so readily be made.

Encouraged by this excellent agreement we then go on to include in our state-averaged approximation scheme further terms from the full SUB2 equations. Thus in Sec. IV we first consider the very important LAD term in SUB2 that generates the particle-particle ladders, and which is vital for a correct treatment of the short-range behavior, in the same sense that the RPA terms are vital for a correct treatment of the long-range behavior. We stress in particular that while it is certainly necessary for a good description at metallic densities to treat both of these limits correctly, this by itself is not sufficient. It is particularly important also to treat carefully the intermediate-range region where the interference of these short- and long-range effects is important. We see in particular that an appreciation of this point forces us even to go beyond the SUB2 approximation and to include some effects of the coupling of the two-body subsystems to three- and four-body subsystems which are im- portant for a proper treatment of the ladder term.

Our final results are presented in Sec. $\mathrm{V}$ after all other terms from the full SUB2 approximation have either been incorporated into our approximate fermion scheme or reasons have been given for their neglect at metallic densities. A comparison of our results in this density regime with other recent essentially exact results (obtained from an exact stochastic simulation of the many-body Schrödinger equation) indicates agreement at the $1 \%$ level, which is at least as good as and probably better than all other microscopic calculations of this system of either the perturbative or variational (or any other) kind. Our final conclusions are presented in Sec. VI.

\section{BOSON RESULTS AND LOW-DENSITY BEHAVIOR}

Before considering the approximations that we shall develop to enable us to handle the Fermi SUB2 equation, it is instructive first to consider the analogous but much simpler equations for a system of bosons in the expectation that a study of the Bose equations may provide us with some insight into the structure of the Fermi equations. In this context it is natural also to consider the lowdensity limit since we expect that in this limit the effect of quantum statistics should become unimportant, i.e., that the energy for a system of bosons should approach that of a system of fermions interacting via the same two-body potential.

Although it seems that a study of the coupled cluster equations for a many-boson system has not previously appeared in the literature, the necessary formalism can be developed in very close analogy to the fermion case, as has been realized many times before. In the following discussion the interested reader may compare with I for the analogous Fermi case and for details concerning the notation. In terms of a suitable model or uncorrelated $N$-body wave funtion $|\Phi\rangle$, the (usual linkedcluster) ansatz for the exact $N$-body ground-state (g.s.) wave function $|\Psi\rangle$,

$$
|\Psi\rangle=e^{S}|\Phi\rangle
$$

is made, and we consider $|\Psi\rangle$ normalized to $|\Phi\rangle$ by $\langle\Phi \mid \Psi\rangle=1$. We consider here only model Fermi states of Slater determinant form

$$
\left|\Phi_{F}\right\rangle=a_{v_{1}}^{\dagger} \cdots a_{v_{N}}^{\dagger}|0\rangle
$$


with $|0\rangle$ the vacuum state, and where the operators $a_{v_{i}}^{\dagger}$ are a set of fermion creation operators for the orthonormalized single-particle (s.p.) states $\left|v_{i}\right\rangle$. For bosons the antisymmetrized product of s.p. states is replaced by the (symmetric) singlestate condensate,

$$
\left|\Phi_{B}\right\rangle=(N !)^{-1 / 2}\left(b_{0}^{\dagger}\right)^{N}|0\rangle,
$$

where the operator $b_{0}^{\dagger}$ creates a boson in state 0 , and, more generally, the operators $b_{\alpha_{i}}^{\dagger}$ create bosons in a complete orthonormal s.p. set $\left|\alpha_{i}\right\rangle$.

Although it is clearly possible to consider more general s.p. states, it is important for later discussions to realize that for all later results reported we henceforth deal exclusively with plane-wave s.p. states. Thus $\left|\Phi_{F}\right\rangle$ represents the usual filled Fermi sea, and $\left|\Phi_{B}\right\rangle$ the usual completely occupied zero-momentum condensate: both isotropic and homogeneous states of zero total momentum. The correlation operator $S$ is decomposed into $n$-body $(n \leq N)$ components, such that in the $N$-particle configuration space we have,

$$
S=\sum_{i<j}^{N} S_{2}(i j)+\sum_{i<j<k}^{N} S_{3}(i j k)+\cdots
$$

where each of the operators $S_{n}\left(i_{1} \cdots i_{n}\right)$ is symmetric in its $n$ arguments. Equivalently, and more conveniently for many purposes, we have in Fock space,

$$
\begin{aligned}
& S=\sum_{n=2}^{N} S_{n}, \\
& S_{n}=\left\{\begin{array}{l}
(n !)^{-1} \sum_{\rho_{1} \cdots \rho_{n}} b_{\rho_{i}}^{\dagger} \cdots b_{\rho_{n}}^{\dagger} S_{n}\left(\rho_{1} \cdots \rho_{n}\right)\left(N^{-1 / 2} b_{0}\right)^{n} \text {, bosons } \\
(n !)^{-2} \sum_{\substack{\rho_{1} \\
v_{1} \cdots \rho_{n}}}^{\sum} a_{\rho_{1}}^{\dagger} \cdots a_{\rho_{n}}^{\dagger}\left\langle\rho_{1} \cdots \rho_{n}\left|S_{n}\right| v_{1} \cdots v_{n}\right\rangle_{A} a_{v_{n}} \cdots a_{v_{1}} \text {, fermions } .
\end{array}\right.
\end{aligned}
$$

Our notation is such that $\left|\alpha_{1} \cdots \alpha_{n}\right\rangle$ represents a direct product state,

$$
\left|\alpha_{1} \cdots \alpha_{n}\right\rangle=\left|\alpha_{1}\right\rangle \cdots\left|\alpha_{n}\right\rangle,
$$

and the subscript $A$ on the ket states for fermions indicates explicit antisymmetrization (without normalization) of that state,

$$
\left|v_{1} \cdots v_{n}\right\rangle_{A}=\sum_{P\{v\}}(-1)^{P_{v}}\left|v_{1} \cdots v_{n}\right\rangle
$$

where the sums extend over all ( $n$ !) permutations of the indices $v_{1} \cdots v_{n}$ and $P_{v}$ is the signature of the permutation. The $n$-body matrix elements of $S_{n}(1 \cdots n)$ for fermions are given in terms of the one-body wave functions $\langle i \mid \alpha\rangle \equiv \phi_{\alpha}(i)$ by

$$
\begin{aligned}
\left\langle\rho_{1} \cdots \rho_{n}\left|S_{n}\right| v_{1} \cdots v_{n}\right\rangle_{A} & =\left\langle\phi_{\rho_{i}}(1) \cdots \phi_{\rho_{n}}(n)\left|S_{n}(1 \cdots n)\right| \sum_{P\{v\}}(-1)^{P_{v}} \phi_{v_{1}}(1) \cdots \phi_{v_{n}}(n)\right\rangle \\
& =\left\langle\phi_{\rho_{1}}(1) \cdots \phi_{\rho_{n}}(n)\left|S_{n}(1 \cdots n)\right| \operatorname{det}_{n}\left\{\phi_{v_{i}}(j)\right\}\right\rangle \\
& =\left\langle(n !)^{-1 / 2} \operatorname{det}_{n}\left\{\phi_{\rho_{i}}(j)\right\}\left|S_{n}(1 \cdots n)\right|(n !)^{-1 / 2} \operatorname{det}_{n}\left\{\phi_{v_{i}}(j)\right\}\right\rangle .
\end{aligned}
$$

As in I we use the convention that the integers in parentheses after an operator whose matrix element is being taken, refer that operator to the quantum labels in the associated bra and ket in the corresponding numerical positions (counting in each case from the left).

The notation employed in Eq. (2.4), and henceforth, reflects the linked-cluster aspect of the ex- pansion, viz., s.p. labels $v_{i}$ indicate states normally occupied in $|\Phi\rangle$ (i.e., states inside the filled Fermi sea for fermions, or the zero-momentum condensate, $v \equiv 0$, for bosons); and s.p. labels $\rho_{i}$ indicate normally unoccupied states. Where necessary later, s.p. labels $\alpha_{i}$ run over all s.p. states (i.e., a complete set). It is important to realize that from the outset we are assuming that $|\Psi\rangle$ and $|\Phi\rangle$ are 
eigenstates of total momentum (with eigenvalue zero), and that the sum in Eq. (2.4a) omits the term $n=1$ ( ince $S_{1} \equiv 0$ ) only as a consequence of this assumption.

We note that whereas in the Fermi case the particles occupy in the state $\left|\Phi_{F}\right\rangle$ all s.p. states up to the Fermi momentum, in the Bose case we assume that only the zero-momentum state is occupied in $\left|\Phi_{B}\right\rangle$. This would seem to imply that our ansatz will only be useful if the true Bose system also has a macroscopic number of particles in the zeromomentum condensate. In general this restrictive assumption need not be made, but we do it here because it simplifies the formalism and because we are interested in the Bose case primarily as a tool to gain a better understanding of the Fermi case.

Physically, $S_{n}$ represents the true correlation operator for an $n$-body subsystem that remains after all the factorizable (or unlinked) correlations have been removed from the (complete) $n$-body subsystem amplitude operator $\Psi_{n}$, defined by its matrix elements,

$$
\begin{aligned}
& \left\langle\alpha_{1} \cdots \alpha_{n}\left|\Psi_{n}\right| v_{1} \cdots v_{n}\right\rangle_{A} \\
& \equiv\left\langle\Phi_{F}\left|a_{v_{1}}^{\dagger} \cdots a_{v_{n}}^{\dagger} a_{\alpha_{n}} \cdots a_{\alpha_{1}}\right| \Psi\right\rangle \text {, fermions } \\
& \Psi_{n}\left(\alpha_{1} \cdots \alpha_{n}\right) \\
& \equiv\left\langle\Phi_{B}\left|\left(N^{-1 / 2} b_{0}^{\dagger}\right)^{n} b_{\alpha_{n}} \cdots b_{\alpha_{1}}\right| \Psi\right\rangle \text {, bosons . }
\end{aligned}
$$

For bosons the subsystem amplitudes $\Psi_{2}$ and $\Psi_{3}$ are given in the thermodynamic limit $(N \rightarrow \infty$, volume $\Omega \rightarrow \infty, \rho=N / \Omega$ finite) by

$$
\begin{aligned}
\Psi_{2}\left(\alpha_{1} \alpha_{2}\right)=( & \left.N^{1 / 2} \delta_{\alpha_{1}, 0}\right)\left(N^{1 / 2} \delta_{\alpha_{2}, 0}\right)+S_{2}\left(\alpha_{1} \alpha_{2}\right) \\
\Psi_{3}\left(\alpha_{1} \alpha_{2} \alpha_{3}\right)= & \left(N^{1 / 2} \delta_{\alpha_{1}, 0}\right)\left(N^{1 / 2} \delta_{\alpha_{2}, 0}\right)\left(N^{1 / 2} \delta_{\alpha_{3}, 0}\right) \\
& +S_{123}\left[S_{2}\left(\alpha_{1} \alpha_{2}\right)\left(N^{1 / 2} \delta_{\alpha_{3}, 0}\right)\right] \\
& +S_{3}\left(\alpha_{1} \alpha_{2} \alpha_{3}\right)
\end{aligned}
$$

where $S_{123}$ generates the sum of all terms obtained by cyclic permutation of the labels $\alpha_{1}, \alpha_{2}$, and $\alpha_{3}$. Our choice of factors of $N^{-1 / 2}$ in Eq. (2.4b) is such that the resulting $S_{n}$ are of order 1, and the factors $N^{1 / 2}$ in Eqs. (2.6) and (2.7) reflect the Bose statistics. For example, Eq. (2.6) gives the amplitude that two particles are in s.p. states $\alpha_{1}$ and $\alpha_{2}$ whereas all other particles remain in the zeromomentum condensate. The Bose statistics then imply that the situation where $\alpha_{1}$ and $\alpha_{2}$ are also zero-momentum states is a factor $N^{2}$ more likely in the thermodynamic limit than the situation that they are in different states. Except for this difference the interpretation of amplitudes and equations is very much the same as that of a fermion system.

An equivalent physical description of $S_{n}$ (for fermions) is that its matrix elements give the exact amplitudes that describe the excitation of $n$ particle-hole pairs; where particles and holes refer, respectively, to states normally unoccupied and normally occupied in the model wave function $|\Phi\rangle$. For bosons the role of the hole states is played by the condensate. It seems intuitively apparent that in order for our ansatz of Eq. (2.1) to be useful, the physical system under consideration ought to share at least qualitatively the features built into the model state $|\Phi\rangle$. More explicitly we expect our choices $\left|\Phi_{F}\right\rangle$ and $\left|\Phi_{B}\right\rangle$ to have relevance, respectively, only to real fermion systems in states in which some semblance of the sharp Fermi surface still remains, and to real Bose systems which contain a finite fraction of the particles in a zero-momentum condensate. This would seem to rule out from the outset for fermions, for example, an accurate description of "abnormal" or "super" phases, or indeed of anything but the usual "liquid" or "Fermi fluid" phase. Later, however, in discussing the low-density results, we give some indication that this intuitive feeling may well be false; or at least that the coupled-cluster formalism may be much more powerful than this too pessimistically narrow interpretation would seem to allow.

The derivation of the coupled-cluster equations for bosons now proceeds in complete analogy with that for fermion systems as discussed in I. The only comments that we make are that if the derivation of Ref. 3 is used it is important to take the thermodynamic limit only at the end of the calculation, and that if the derivation of Ref. 4 is used one has to shift operators in an appropriate manner since Wick's theorem can no longer be used. For spin-zero bosons, the exact two-body equation for the quantity

$$
S_{2}(q) \equiv S_{2}(\overrightarrow{\mathrm{q}},-\overrightarrow{\mathrm{q}})
$$

is readily found to be given in the thermodynamic limit by

$$
\begin{aligned}
& \frac{\hbar^{2} q^{2}}{m} S_{2}(q)+T_{\mathrm{RPA}}+T_{\mathrm{CP}}+T_{\mathrm{LAD}} \\
& +\sum_{\overrightarrow{\mathrm{q}}^{\prime}}\left[2 N^{1 / 2} S_{3}\left(\overrightarrow{\mathrm{q}}, \overrightarrow{\mathrm{q}}^{\prime},-\overrightarrow{\mathrm{q}}-\overrightarrow{\mathrm{q}}^{\prime}\right)\right. \\
& \left.\quad+\frac{1}{2} N S_{4}\left(\overrightarrow{\mathrm{q}},-\overrightarrow{\mathrm{q}}, \overrightarrow{\mathrm{q}}^{\prime},-\overrightarrow{\mathrm{q}}^{\prime}\right)\right] V\left(q^{\prime}\right)=0,
\end{aligned}
$$

where $m$ is the mass of each particle, and 


$$
\begin{aligned}
& T_{\mathrm{RPA}}=N V(q)\left[1+S_{2}(q)\right]^{2}, \\
& T_{\mathrm{CP}}=-4 \frac{E}{N} S_{2}(q), \\
& T_{\mathrm{LAD}}=\sum_{\overrightarrow{\mathrm{q}}^{\prime}} V\left(\overrightarrow{\mathrm{q}}-\overrightarrow{\mathrm{q}}^{\prime}\right) S_{2}\left(q^{\prime}\right),
\end{aligned}
$$

and where the ground-state (g.s.) energy per particle is given by

$$
E / N=\frac{1}{2} N V(0)+\frac{1}{2} \sum_{\overrightarrow{\mathrm{q}}} V(q) S_{2}(q) .
$$

We are interested in Eq. (2.9) only in the SUB2 approximation which is obtained from it by setting $S_{3}$ and $S_{4}$ to zero. This truncated equation may then be compared with its analog for Fermi systems which appears in I as Eq. (2.15). It is immediately apparent that not only are there far fewer terms in the Bose equation but that the individual terms are much less complex. In Fig. 1, we represent the various terms of the Bose SUB2 equation in the same way as was done in $I$ for the Fermi case. The terms labeled RPA of Eq. (2.10) are represented pictorially by diagrams 1 (a) -1 (c) of Fig. 1, and it is again clear that these generate by themselves the ring or bubble diagrams of the RPA. The Bose RPA diagrams are formally identical to diagrams 1 (a) -1 (c) of Fig. 1 in $I$ in the Fermi case. The terms labeled CP (condensate potential) are shown in diagrams $1(\mathrm{~d})$ and 1(e) of Fig. 1 , and represent the self-consistent energy insertions on the zero-momentum condensate lines; i.e., the (self-consistent) condensate potential. These terms are the analog of the complete hole-potential

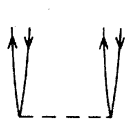

(a)

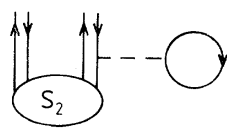

(d)

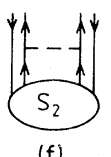

FIG. 1. Diagrammatic representation of the various terms in the boson SUB2 equation. Note that the asymmetric diagrams (b), (d), and (e) each represent two separate terms; one as shown and another obtained by mirror reflection.
(CHP) terms in the Fermi case, discussed in I. Finally, diagram 1(f) of Fig. 1 represents the LAD term that is responsible for scattering two particles outside the condensate and which hence generates the two-particle ladder (LAD) diagrams. This term also occurred in the Fermi case in I as part of the complete ladder (CLAD) contribution, where it was represented as diagram $(m)$ of Fig. 1.

In the Fermi case it was a nontrivial problem to derive the already well-known high-density (RPA) results, and indeed the major part of paper I was concerned with this derivation. In marked contrast, the simplicity of the Bose equations allows us at once to deal with both the high- and low-density limits, and we discuss these now for the one-component Coulomb plasma. The Coulomb potential with a uniform, rigid, and neutralizing background present is

$$
V(q)=\frac{4 \pi e^{2}}{\Omega q^{2}}\left(1-\delta_{\overrightarrow{\mathrm{q}}, 0}\right) .
$$

The number density $\rho$ may be expressed either in terms of the usual dimensionless coupling constant $r_{s}$, which is the average interparticle spacing in units of the Bohr radius $a_{0} \equiv \hbar^{2} / m e^{2}$, or in terms of a (for bosons, purely fictitious) Fermi wave number $k_{F}$ applicable to an unpolarized spin- $\frac{1}{2}$ system,

$$
\rho=\left(4 \pi r_{s}^{3} a_{0}^{3} / 3\right)^{-1}=k_{F}^{3} / 3 \pi^{2},
$$

and which is defined for ease of comparison with the electron plasma. Henceforth the ground-state (g.s.) energy per particle is expressed in Rydberg units

$$
E / N=\epsilon\left(e^{2} / 2 a_{0}\right),
$$

and any dimensionless momentum variables that appear have been scaled against the Fermi momentum $\hbar k_{F}$ defined by Eq. (2.15).

Inserting the potential from Eq. (2.14) into the Bose SUB2 equation gives, in dimensionless variables and in the thermodynamic limit,

$$
\begin{gathered}
q^{2} S_{2}(q)+4 \alpha r_{s}\left(3 \pi q^{2}\right)^{-1}\left[1+S_{2}(q)\right]^{2}-2\left(\alpha r_{s}\right)^{2} \epsilon S_{2}(q) \\
+\frac{\alpha r_{s}}{\pi q} \int_{0}^{\infty} d q^{\prime} q^{\prime} \ln \left|\frac{q^{\prime}+q}{q^{\prime}-q}\right| S_{2}\left(q^{\prime}\right)=0
\end{gathered}
$$

where the energy is given by

$$
\begin{aligned}
\epsilon & =2\left(\pi \alpha r_{s}\right)^{-1} \int_{0}^{\infty} d q S_{2}(q) \\
& \equiv \int_{0}^{\infty} d q \epsilon(q)
\end{aligned}
$$


and where the parameter $\alpha$ is defined as usual by,

$$
a_{0} k_{F}=\left(\alpha r_{s}\right)^{-1}
$$

which implies

$$
\alpha=(9 \pi / 4)^{-1 / 3} \text {. }
$$

The four terms in Eq. (2.17) represent, respectively, the kinetic energy (KE), RPA, CP, and LAD contributions. Although Eqs. (2.17) and (2.18) are readily amenable to numerical solution it is more instructive here to examine them in the highdensity $\left(r_{s} \rightarrow 0\right)$ and low-density $\left(r_{s} \rightarrow \infty\right)$ limits. For a preliminary discussion we neglect the LAD term and discuss its influence briefly at the end of this section. The remaining quadratic equation for $S_{2}$ is trivially solved to give, in this SUB2-LAD approximation,

$$
\begin{aligned}
S_{2}(q)= & -1-\frac{3 \pi q^{2}}{8 \alpha r_{s}}\left[q^{2}-2\left(\alpha r_{s}\right)^{2} \epsilon\right] \\
& +\left[\left[1+\frac{3 \pi q^{2}}{8 \alpha r_{s}}\left[q^{2}-2\left(\alpha r_{s}\right)^{2} \epsilon\right]\right]^{2}-1\right]^{1 / 2},
\end{aligned}
$$

where the negative square root has to be discarded since it gives an unbounded solution $\left[S_{2}(q) \rightarrow \infty\right.$ as $q \rightarrow \infty]$.

In the high-density limit it is readily shown that to leading order for the energy only the KE and RPA terms contribute, and we find trivially,

$$
\underset{r_{s} \rightarrow 0}{\epsilon \rightarrow \mathrm{RPA}}=Q_{\mathrm{RPA}} r_{s}^{-3 / 4}
$$

where the constant $Q_{\mathrm{RPA}}$ is given by

$$
\begin{aligned}
Q_{\mathrm{RPA}} & \equiv Q \\
& =-2 \pi^{-1}\left(6^{1 / 4}\right) \int_{0}^{\infty} d x\left[1+x^{4}-x^{2}\left(x^{4}+2\right)^{1 / 2}\right] \\
& =-\frac{16}{5}\left(\frac{3}{\pi^{2}}\right)^{1 / 4} \frac{\Gamma\left(\frac{3}{4}\right)}{\Gamma\left(\frac{1}{4}\right)} \\
& \approx-0.8031
\end{aligned}
$$

which is the exact result first obtained by Foldy. ${ }^{5}$ We note also for comparison purposes with the Fermi case, that the Bose equations may also be solved in this limit in the TDA. Just as in I the TDA is obtained from the RPA by ignoring the term quadratic in $S_{2}$. We find

$$
\begin{aligned}
& \epsilon_{\mathrm{TDA}}=Q_{\mathrm{TDA}} r_{s}^{-3 / 4}, \\
& Q_{\mathrm{TDA}}=-\left(\frac{3}{32}\right)^{1 / 4} \approx-0.5533 .
\end{aligned}
$$

It is also not difficult to show that the next term in the high-density Bose expansion is a constant,

$$
\underset{r_{s} \rightarrow 0}{\epsilon \rightarrow} Q r_{s}^{-3 / 4}+R
$$

and that both the CP and LAD terms in Eq. (2.17) now also contribute to $R$. We find

$$
\begin{aligned}
& R_{\mathrm{SUB} 2}=R_{\mathrm{CP}}+R_{\mathrm{LAD}}, \\
& R_{\mathrm{CP}}=\frac{16}{15 \pi}, \quad R_{\mathrm{LAD}}=\frac{32}{45 \pi} .
\end{aligned}
$$

By inspecting Eq. (2.9) and the equivalent relations for $S_{3}$ and $S_{4}$ it can, however, be shown that the coupling terms to $S_{3}$ and $S_{4}$ in the exact Eq. (2.9) also contribute to the constant $R$ (although not to $Q$ ) in Eq. (2.25). We have also calculated the constant $R$ given by the exact two-body Eq. (2.9), keeping the complete coupling to three- and fourbody clusters. To this order we find, for example, that the three-body correlation amplitude $S_{3}$ needed in Eq. (2.9) may be replaced by

$$
-N^{1 / 2} S_{3}\left(\overrightarrow{\mathrm{q}}_{1}, \overrightarrow{\mathrm{q}}_{2}, \overrightarrow{\mathrm{q}}_{3}\right) \rightarrow\left[\omega\left(q_{1}\right)+\omega\left(q_{2}\right)+\omega\left(q_{3}\right)\right]^{-1} S_{123}\left(\left\{V\left(q_{1}\right)\left[1+S_{2}\left(q_{1}\right)\right]+V\left(q_{2}\right)\left[1+S_{2}\left(q_{2}\right)\right]\right\} S_{2}\left(q_{3}\right)\right)
$$

for $\left(\vec{q}_{1}+\vec{q}_{2}+\vec{q}_{3}\right)=0$, and where the effective s.p. energy is

$$
\omega(q)=\hbar^{2} q^{2} / 2 m+N V(q)\left[1+S_{2}(q)\right] .
$$

A similar replacement may also be made for $S_{4}$ by examining the four-body equation. Equations (2.9) - (2.13) then lead to

$$
R=R_{\mathrm{SUB} 2}+R_{3}+R_{4} \approx 0.0280,
$$

where both the contributions $R_{3}$ and $R_{4}$, from the coupling terms to $S_{3}$ and $S_{4}$, respectively, in Eq. (2.9), are finite. The final result of Eq. (2.29) is ex$a c t$, and an extremely tedious rearrangement of the integrations shows it to be in precise agreement with the first correct result reported of Brueckner. ${ }^{6}$ It is worth pointing out here that by contrast with most competing methods, each of the terms in Eqs. (2.26) and (2.29) is finite, and no cancellation of spurious logarithmic singularities occurs. This particular point highlights a more general advan- 
tage of the coupled-cluster formalism-namely that terms which tend to cancel each other are automatically grouped together (or never split apart).

Turning now to the much more revealing lowdensity limit, naively one would not expect the SUB2 approximation or Eq. (2.21) to give any reasonable result at all in this strong-coupling regime, since one imagines that the $n$-body clusters even with $n \gg 2$ are still very important. Indeed we believe that the real Coulomb system undergoes a phase transition to a Wigner solid ${ }^{7}$ in this limit, and the solid may be thought of as an archetypal system where the $N$-body correlations dominate. At any rate it is clear that the low-density Coulomb systems provide one of the most stringent tests for our formalism.

It can readily be shown from Eqs. (2.9) - (2.13) that in the SUB2 approximation we have

$$
\epsilon_{\mathrm{SUB} 2} \underset{r_{s} \rightarrow \infty}{\rightarrow}-A r_{s}^{-1}+B r_{s}^{-3 / 2}+O\left(r_{s}^{2}\right),
$$

where the KE term contributes only to the constant $B$ in leading order. In this limit the terms RPA, CP, and LAD are all necessary for a quantitative evaluation of the constant $A$, but they play distinctly different qualitative roles. Thus, it is vital to keep the RPA terms to get the correct analytic behavior because, as expected, these terms continue to be crucial for the long-range $(q \rightarrow 0)$ screening of the Coulomb potential. Similarly the CP plays a crucial role now at higher values of $q$. Whereas the inclusion of the LAD term quantitatively changes the constants $A$ and $B$ in Eq. (2.30), it may safely be omitted without changing the analytic form. Dropping the LAD term, and hence using Eq. (2.21), we find for the SUB2 - LAD approximation:

$$
\begin{aligned}
& A=\left(32 / 3 \pi^{2}\right)^{1 / 3} \approx 1.03, \\
& B=3^{1 / 2} \pi / 8 \approx 0.68 .
\end{aligned}
$$

We have thus obtained a g.s. energy in the lowdensity limit which to leading order seems to be purely potential energy. This may be verified by using the following exact relations for a system interacting via two-body Coulomb forces,

$$
\begin{aligned}
& \bar{t}=-\frac{d\left(r_{s} \epsilon\right)}{d r_{s}}, \\
& \bar{v}=\frac{1}{r_{s}} \frac{d\left(r_{s}^{2} \epsilon\right)}{d r_{s}},
\end{aligned}
$$

where $\bar{t}$ and $\bar{v}$ are the expectation values of the kinetic and potential energy per particle, respectively, each expressed in Rydberg units. The relations (2.32) may be derived either from the quantum virial theorem or by using the well-known theorem attributed to Pauli, ${ }^{8}$ which connects the energy with an integral of the potential energy over the coupling constant. Use of Eqs. (2.30) and (2.32) furthermore shows that the much more interesting second term in Eq. (2.30) is exactly one-half each kinetic and potential energy - which at the very least is strongly reminiscent of simple harmonic motion and the behavior expected of a solid.

It is well known that in the low-density limit the kinetic energy of the particles becomes negligibly small, and the Coulomb interactions dominate in determining the many-body wave function. As first pointed out by Wigner, ${ }^{7}$ the energy of the system in this situation should be minimized by the particles arranging themselves in a regular periodic lattice. This then leads to an energy proportional to $r_{s}^{-1}$. Whereas in a fluid phase the particles are free to occupy the whole volume, which by the uncertainty principle leads to a kinetic energy proportional to $r_{s}^{-2}$, in the Wigner solid phase the particles are constrained to oscillate about the fixed lattice sites and hence to have a greater kinetic energy. Elementary considerations of simple harmonic motion show that the volume available to the particles is a fraction proportional to $\left(r_{s}^{3 / 4} / r_{s}\right)^{3}=r_{s}^{-3 / 4}$ of the total volume. Hence by the uncertainty principle the kinetic energy is increased by a fraction proportional to $\left(r_{s}^{3 / 4} / r_{s}\right)^{-2}=r_{s}^{1 / 2}$, thus leading to an energy of zero-point motion proportional to $r_{s}^{-3 / 2}$. As this Wigner model suggests, the exact expansion for the energy in the low-density limit is a power series in $r_{s}^{-1 / 2}$ where the terms of order $r_{s}^{-2}$ and higher are due to anharmonicities in the zero-point motion. Based on a bcc lattice, Carr et al. ${ }^{9}$ give

$$
\begin{gathered}
\epsilon_{\text {exact }} \underset{r_{s} \rightarrow \infty}{\rightarrow}-1.792 r_{s}^{-1}+2.65 r_{s}^{-3 / 2} \\
-0.73 r_{s}^{-2}+\cdots .
\end{gathered}
$$

It is clear that our approximation in Eqs. (2.30) and (2.31) has the correct analytic form for the energy of the solid, although the values of the coefficients are considerably underestimated. What is more important, however, is that even the lowest (SUB2) approximation in the coupled-cluster scheme gives a low-density energy which cannot possibly represent what is normally understood by a fluid phase, since the particles are definitely not free to occupy the whole volume. What is perhaps most difficult to visualize is how our intrinsically 
"fluidlike" and everywhere translationally-invariant approach can really provide such a good description of the solid phase, as well as the fluid phase. We attempt to understand this by similar reasoning to that behind the familiar "floating crystal" model of Feenberg. ${ }^{10}$ Thus, while one usually describes a solid in terms of a fixed set of lattice sites with respect to some fixed external reference frame, one can just as well specify a description in which the wave function obtained from the former description is averaged with respect both to all possible orientations of the symmetry axes and to all translations of the lattice within range of the unit cell. This latter description is, of course, translationally invariant, and while all information is lost on the positions of the lattice sites and on the orientations of the symmetry axes, it nevertheless retains complete information on the internal structure. In our SUB2 approximation, however, all this information is contained in the amplitude $S_{2}$ which is related by Eq. (2.6) to the two-body subsystem amplitude $\Psi_{2}$. We pointed out already in I the restrictions which are implied by describing a many-body system in terms of such an amplitude. Clearly three-body and higher cluster effects are treated in an average sense only, and hence we cannot expect to represent the real detailed internal structure of a crystal-lattice wave function. What our SUB2 approximation is describing in the lowdensity limit must be some sort of amorphous solid rather than a real crystal. We can perhaps most appropriately describe our approximation of this limit by claiming that our jellium has set.

While we have presented explicit evidence that this neglect of higher-order correlations is not a severe limitation at least as far as the qualitative behavior of the g.s. energy is concerned (and probably also for matrix elements of some other fewbody operators), the fact remains that for a charged Bose system in the low-density limit, the third- and higher-order correlations are still very strong. Thus, more accurate values of the coefficients $A$ and $B$ in Eq. (2.30), in comparison with those of Eq. (2.33), simply cannot be expected. We note here also that preliminary numerical calculations of the complete SUB2 equations (2.17) and (2.18), including the LAD term, do not change this overall picture. Inclusion of the LAD term gives a monotonically increasing repulsive contribution to the g.s. energy as a function of $r_{s}$, starting from zero at $r_{s}=0$ and giving about a $20 \%$ reduction in binding at $r_{s} \rightarrow \infty$, thereby increasing the discrepancy with the Wigner value. (We also note, how- ever, that an evaluation of the two-body radial distribution function within the SUB2 approximation gives a positive-definite function at all densities only so long as the LAD term is included.) Finally we note that although the SUB2 approximation (and even the algebraic quadratic equation approximation of SUB2-LAD) works superbly over the entire density regime for the Bose Coulomb plas$\mathrm{ma}$, the g.s. energy is quantitatively unsatisfactory in the low-density limit. It is clear that higherorder clusters must be incorporated for quantitatively accurate results in this limit, but due to the relative simplicity of the Bose coupled-cluster equations this is quite practicable, as indeed we have already indicated in the high-density limit. Since we are primarily interested here in the electron plasma results, however, we now leave the Bose problem, and we indicate in the next section how the experience obtained in this case can be applied to the Fermi problem.

To conclude this section we discuss briefly the low-density limit in the case of an electron plasma. The situation for fermions is much more favorable in this limit than for bosons, in one sense, since the Pauli principle very effectively hinders electrons from clustering in groups of more than two, thus forcing the higher correlations to be smaller, keeping particles apart from each other, and making the system more "solidlike" in lower levels of approximation. Although in the exact Wigner lowdensity limit the effects of quantum statistics vanish, with the fermion and boson solid both described by the same asymptotic expansion (2.33) (and

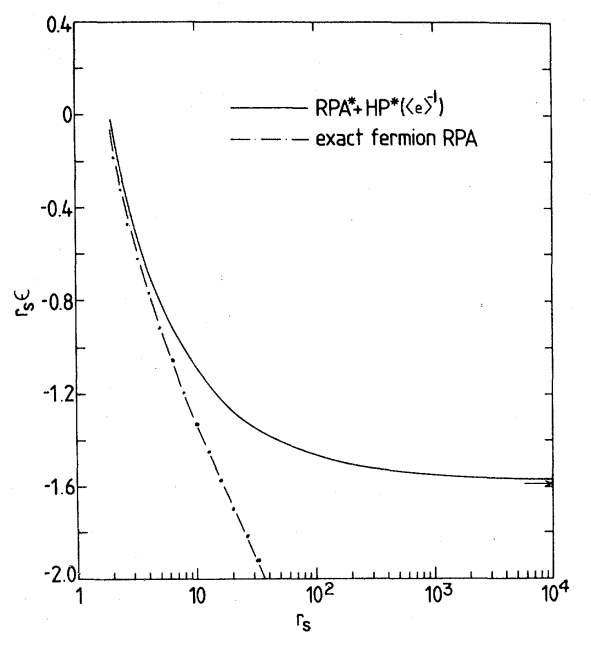

FIG. 2. Fermion ground-state energy per article, $\epsilon$, shown as $\left(r_{s} \epsilon\right)$ vs $r_{s}$ in two different approximations: the $\mathrm{RPA}^{*}+\mathrm{HP}^{*}\left(\langle e\rangle^{-1}\right)$ approximation of Eq. (2.34), and $-\cdot-\cdot-$ exact RPA. 
the different statistics reflected only in differing terms which vanish exponentially with $\left.r_{s} \rightarrow \infty\right),{ }^{9}$ this is by no means true in our translationallyinvariant coupled-cluster description. In the case of the electron system, exchange terms do not vanish and the convergence of the coupled-cluster hierarchy is thereby much improved from the Bose case.

Thus, for example, the fermion equation analogous to the SUB2-LAD approximation described above for bosons, is obtained from Eq. (2.15) of I by keeping the RPA and hole-potential (HP) terms only (apart from the kinetic energy terms). Applying a particular approximation to this resulting equation, which will be fully developed in the remaining sections, we obtain the "averaged" Fermi analog of the Bose SUB2-LAD approximation,

$\overline{S_{2}}(q)=-\frac{4}{3 \pi}\left(\alpha r_{s}\right) \frac{1}{q^{2} \widetilde{e}} P^{2}(q)\left(1+\frac{\bar{S}_{2}(q)}{P(q)}\right)^{2}$,

$\widetilde{e}=\langle e\rangle-\frac{4}{\pi}\left(\alpha r_{s}\right) \int_{0}^{\infty} d q^{\prime} \frac{P\left(q, q^{\prime}\right)}{P\left(q^{\prime}\right) P(q)} \bar{S}_{2}\left(q^{\prime}\right)$.

The approximation, as described in Sec. III, basically involves averaging over momenta inside the Fermi sea, and $\bar{S}_{2}$ and $\langle e\rangle$ are known functions which arise, respectively, from thus state-averaging the two-particle-two-hole amplitude $S_{2}$ and its associated kinetic energy expression in Eq. (2.15) of I. The functions $P(q)$ and $P\left(q, q^{\prime}\right)$ that also arise are explicitly defined and discussed later in Eqs. (3.16) and (4.14). For fermions, the g.s. energy is given by Eqs. (3.5) and (3.6) of I, and it is now the correlation energy that is given by Eq. (2.18) [with $S_{2}(q)$ replaced by $\bar{S}_{2}(q)$ ]. We give reasons below that enable us to expect that the averaging procedure implied by Eq. (2.34) is accurate to a few percent for the g.s. energy $\epsilon$. In Fig. 2 we show the results obtained for the quantity $\left(\epsilon r_{s}\right)$ as a function of $r_{s}$. A very precise numerical solution of Eq. (2.34), accurate to seven significant figures, showed that the energy has the same low-density analytic expansion (2.30), and that the values of the coefficients are

Fermi (RPA + HP-averaged approx.):

$$
A \approx 1.5845, B \approx 1.10 \text {. }
$$

The value of the leading coefficient $A$ is thus seen to be in much better agreement with the exact Wigner result (2.33) than for the analogous Bose result (2.31).

In the remainder of this paper we now discuss the application of the coupled-cluster equations (in SUB2 approximation and beyond) developed in I, to the electron plasma, with particular emphasis on the metallic-density or intermediate-coupling $\left(1 \lesssim r_{s} \lesssim 5\right)$ regime.

\section{THE FERMION APPROXIMATION SCHEME AND APPLICATIONS TO RPA AND RPA-EXCHANGE EFFECTS}

Armed with the results obtained in Sec. II for bosons we now return to our exact Fermi SUB2 description defined in detail in I, and attempt to develop a consistent set of approximations that will enable us to study in detail the structure of the equations and which will lead hopefully to an accurate description of the one-component electron plasma over the entire density range. The SUB2 equations for fermions, although conceptually similar to those for bosons, are mathematically vastly more complex due both to the many more terms required by antisymmetrization, and to the state- (i.e., momentum-) dependence induced by the hole states inside the Fermi sea in comparison with the unique zero-momentum condensate for bosons. In particular, for electrons the matrix elements

$$
\begin{aligned}
& S_{2 ; \overrightarrow{\mathrm{k}}_{1} \overrightarrow{\mathrm{k}}_{2}}^{\sigma_{1} \sigma_{2}}(\overrightarrow{\mathrm{q}}) \\
& \quad \equiv\left\langle\overrightarrow{\mathrm{k}}_{1}+\overrightarrow{\mathrm{q}}, \sigma_{1} ; \overrightarrow{\mathrm{k}}_{2}-\overrightarrow{\mathrm{q}}, \sigma_{2}\left|S_{2}\right| \overrightarrow{\mathrm{k}}_{1} \sigma_{1} ; \overrightarrow{\mathrm{k}}_{2} \sigma_{2}\right\rangle_{A},
\end{aligned}
$$

depend not only on a momentum transfer $\vec{q}$ as for bosons [and see Eq. (2.8)] but also on the two hole momenta $\overrightarrow{\mathrm{k}}_{1}$ and $\overrightarrow{\mathrm{k}}_{2}$, as well as the (up or down) spin indices $\sigma_{1}$ and $\sigma_{2}$. The complete SUB2 equation for charged Fermi systems has been discussed in detail in I, and it is clear that a numerical solution of this nonlinear integral equation for a function of three three-vectors, while perhaps just feasible with the most powerful modern computers, is not to be undertaken lightly. Accordingly we examine various limits and approximation schemes for handling the coupled-cluster Fermi equations, and it is these we now examine.

In the high-density limit, the RPA again gives the leading contribution to the correlation energy $\epsilon_{c}$, i.e., the g.s. energy relative to the (uncorrelated) Hartree-Fock energy. In I the nonlinear integral equation for $S_{2}$ in RPA was solved exactly and in some detail, both confirming the well-known results of Gell-Mann and Brueckner, ${ }^{11}$ and giving for the first time exact analytic forms for the fourpoint function $S_{2}$ and the once-integrated three- 
point particle-hole vertex function. The TammDancoff approximation (TDA) to the ring summation was also formulated, and the analogous exact solutions in TDA were also presented for the electron gas for the first time.

Turning to the intermediate-coupling $\left(1 \lesssim r_{s} \lesssim 5\right)$ metallic-density regime, we no longer expect the RPA plus second-order exchange to be a good approximation, although it gives the first two terms in the high-density expansion for $\epsilon_{c}$ exactly. Thus, quite apart from ignoring (a) the simple exchange effects necessary to antisymmetrize RPA, we have ignored even in SUB2 approximation: (b) all of the combined particle-particle and hole-hole ladder terms, some at least of which are important for the correct short-range behavior, (c) the generalized self-energy correction terms which self-consistently generate both the particle potential and, much more importantly, the hole potential (which now for fermions plays a similar crucial role to the $\mathbf{C P}$ for bosons), (d) classes of higher ring-exchange terms, and (e) a class of additional exchange terms which includes the particle-hole ladder terms. These terms have all been described in Sec. II of I, and in order systematically to deal with these effects we now propose and implement a further approximation scheme that enables us to study these terms much more readily.

Based on the comparison with Bose systems, the fermion equations should be much simpler if they could be "state averaged," and the basic approximation is thus to average over the initial hole momenta $\overrightarrow{\mathrm{k}}_{1}$ and $\overrightarrow{\mathrm{k}}_{2}$ in the elements of Eq. (3.1) but to keep the important exact property that particle states $\left(\overrightarrow{\mathrm{k}}_{1}+\overrightarrow{\mathrm{q}}\right),\left(\overrightarrow{\mathrm{k}}_{2}-\overrightarrow{\mathrm{q}}\right)$ lie outside the Fermi sea (i.e., the Pauli principle is implemented exactly). In this way the exact $S_{2 ; \overrightarrow{\mathrm{k}}_{1} \overrightarrow{\mathrm{k}}_{2}}(\overrightarrow{\mathrm{q}})$ is replaced by an averaged $\left\langle S_{2}(q)\right\rangle$ and the resulting coupled-cluster equation considered then still has to be state averaged. Although the procedure for this latter step is not unique, this works to our advantage for two reasons: (i) the averaging can be made on physically motivated grounds rather than being imposed arbitrarily, and (ii) since we know exact results for $S_{2}$ in at least one limit, namely the RPA and TDA results for $r_{s} \rightarrow 0$, the errors induced by the various averaging schemes can be checked. Before describ- ing the averaging schemes used in detail, consider as an illustration carrying out the above procedure in RPA. This leads to an equation for $S_{2}$ which involves only the kinetic energy (KE) and RPA terms. After the replacement $S_{2} \rightarrow\left\langle S_{2}\right\rangle$ has been made the only state dependence left is in the KE term, which for fermions is given by the first term of Eq. (2.15) of I, and is proportional to

$\frac{1}{2}\left(\left|\overrightarrow{\mathrm{k}}_{1}+\overrightarrow{\mathrm{q}}\right|^{2}+\left|\overrightarrow{\mathrm{k}}_{2}-\overrightarrow{\mathrm{q}}\right|^{2}-k_{1}^{2}-k_{2}^{2}\right) S_{2} \equiv e S_{2}$.

As two obvious averaging schemes one could imagine (a) replacing $e \rightarrow\langle e\rangle$, or (b) the intuitively and physically more appealing idea of first dividing through by $e$ and then averaging the "energy denominator" $e^{-1} \rightarrow\left\langle e^{-1}\right\rangle$. Both of these schemes are considered in detail below. We show that the former procedure leads precisely to the mean spherical approximation (which is discussed by Zabolitzky ${ }^{12}$ for the electron gas, and which his state-independent, variational, Fermi hypernetted chain (FHNC) formalism leads to in this $r_{s} \rightarrow 0$ limit), which gives an $\epsilon_{c}$ in error by $8.4 \%$ at $r_{s} \rightarrow 0$. The latter procedure, on the other hand, is exact at $r_{s} \rightarrow 0$.

In order to describe our averaging schemes in detail it is probably easier and more comprehensible to perform the necessary approximations individually on the various SUB2 terms. In the remainder of this section we deal with a specific approximation to the fermion SUB2 equation and discuss its exact (numerical) solution. We then describe in detail our approximation scheme which is basic for the remainder of this paper, and compare the results obtained with it to the exact solutions. In Secs. IV and V we then develop the approximation to the full SUB2 scheme (and beyond).

The initial subapproximation to the SUB2 equation that we consider is essentially the lowest-order one which really makes any sense for the electron plasma at metallic densities, viz., keeping only the RPA and RPAEX terms in Eq. (2.15) of I (apart, of course, from the KE term). Measuring all momenta in units of $\hbar k_{F}$, which is the convention that we henceforth consistently adopt, Eqs. (2.15) - (2.20) of I then give us the following coupled equations for the functions $S_{2}^{\dagger \downarrow}\left(\equiv S_{2}^{\downarrow \uparrow}\right)$ and $S_{2}^{\dagger \uparrow}\left(\equiv S_{2}^{\downarrow \downarrow}\right)$ in this RPA + RPAEX scheme:

$$
S_{2 ; \overrightarrow{\mathrm{k}}_{1} \overrightarrow{\mathrm{k}}_{2}}^{\uparrow \downarrow}(\overrightarrow{\mathrm{q}})=-\left(\alpha r_{s}\right)\left(\frac{4}{3 \pi N}\right)\left(\frac{1}{e} \int \frac{1}{q^{2}}\left[1+2 f_{\overrightarrow{\mathrm{k}}_{1}}(\overrightarrow{\mathrm{q}})\right]\left[1+2 f_{-\overrightarrow{\mathrm{k}}_{2}}(\overrightarrow{\mathrm{q}})\right],\right.
$$




$$
\begin{aligned}
& S_{2 ; \overrightarrow{\mathrm{k}}_{1} \overrightarrow{\mathrm{k}}_{2}}(\overrightarrow{\mathrm{q}})=-\left(\alpha r_{s}\right)\left(\frac{4}{3 \pi N}\right) \frac{1}{e}\left\{q^{-2}\left[1+2 f_{\overrightarrow{\mathrm{k}}_{1}}(\overrightarrow{\mathrm{q}})\right]\left[1+2 f_{-\overrightarrow{\mathrm{k}}_{2}}(\overrightarrow{\mathrm{q}})\right]\right. \\
& \left.-q_{\mathrm{ex}}^{-2}\left[1+2 f_{\overrightarrow{\mathrm{k}}_{1}}\left(\overrightarrow{\mathrm{q}}_{\mathrm{ex}}\right)\right]\left[1+2 f_{-\overrightarrow{\mathrm{k}}_{2}}\left(\overrightarrow{\mathrm{q}}_{\mathrm{ex}}\right)\right]\right\} \\
& f_{\overrightarrow{\mathrm{k}}_{1}}(\overrightarrow{\mathrm{q}})=\sum_{\overrightarrow{\mathrm{k}}_{2}} S_{2 ; \overrightarrow{\mathrm{k}}_{1} \overrightarrow{\mathrm{k}}_{2}}(\overrightarrow{\mathrm{q}}) \Theta\left(-\overrightarrow{\mathrm{k}}_{2}, \overrightarrow{\mathrm{q}}\right)
\end{aligned}
$$

and where the following definitions have been used,

$$
\begin{aligned}
& S_{2 ; \overrightarrow{\mathrm{k}}_{1} \overrightarrow{\mathrm{k}}_{2}}(\overrightarrow{\mathrm{q}}) \equiv \frac{1}{2}\left[S_{2 ; \overrightarrow{\mathrm{k}}_{1} \overrightarrow{\mathrm{k}}_{2}}^{\dagger \downarrow}(\overrightarrow{\mathrm{q}})+S_{2 ; \overrightarrow{\mathrm{k}}_{1} \overrightarrow{\mathrm{k}}_{2}}^{\dagger \uparrow}(\overrightarrow{\mathrm{q}})\right], \\
& \Theta(\overrightarrow{\mathrm{k}}, \overrightarrow{\mathrm{q}}) \equiv \Theta(1-k) \Theta(|\overrightarrow{\mathrm{k}}+\overrightarrow{\mathrm{q}}|-1),
\end{aligned}
$$

where $\theta(x)$ is the usual unit step function defined to be one (zero) for $x$ greater (less) than zero,

$$
\begin{aligned}
e & \equiv \frac{1}{2}\left[\left|\overrightarrow{\mathrm{k}}_{1}+\overrightarrow{\mathrm{q}}\right|^{2}+\left|\overrightarrow{\mathrm{k}}_{2}-\overrightarrow{\mathrm{q}}\right|^{2}-k_{1}^{2}-k_{2}^{2}\right] \\
& =\overrightarrow{\mathrm{q}} \cdot\left(\overrightarrow{\mathrm{k}}_{1}-\overrightarrow{\mathrm{k}}_{2}+\overrightarrow{\mathrm{q}}\right)
\end{aligned}
$$

and

$$
\overrightarrow{\mathrm{q}}_{\mathrm{ex}} \equiv \overrightarrow{\mathrm{k}}_{2}-\overrightarrow{\mathrm{k}}_{1}-\overrightarrow{\mathrm{q}}
$$

We note that the RPA + RPAEX equations already display practically the full complexity of the complete SUB2 equations. In the remaining sections we will only add more terms to the righthand sides of Eqs. (3.2) and (3.3). We note also that we reobtain the RPA equations discussed fully in I simply by ignoring the second (exchange) term on the right-hand side of Eq. (3.3), which is just the RPAEX term of I.

The analytic solution obtained in I for the RPA was made possible only because the RPA equation could be reduced to a one-dimensional integral equation. The introduction of the RPAEX terms destroys this simplicity through the introduction of the momentum transfer $\vec{q}_{e x}$, which in turn introduces a complex dependence on the relative orientations of the vectors $\overrightarrow{\mathrm{k}}_{1}, \overrightarrow{\mathrm{k}}_{2}$, and $\overrightarrow{\mathrm{q}}$ which was previously absent. In fact, even a numerical solution of the set of Eqs. (3.2) - (3.4) would not be easy. Indeed Freeman, ${ }^{13}$ who was concerned with such a numerical solution of the coupled-cluster equations (including exchange), in fact only computed an exchange energy which is obtained from Eqs. (3.2) - (3.4), not by a full solution, but merely by iterating them once only starting from the RPA solution. Inserting the result of this single iteration into the exact expression for the correlation energy [c.f. Eq. (3.7) of I],

$$
\begin{aligned}
\epsilon_{c} & \left.=\int \frac{1}{2 N}\right]\left[\frac{1}{\alpha r_{s}}\right]\left[\frac{8}{\pi}\right] \int_{0}^{\infty} d q \sum_{\overrightarrow{\mathrm{k}}_{1} \overrightarrow{\mathrm{k}}_{2}}\left[S_{2 ; \overrightarrow{\mathrm{k}}_{1} \overrightarrow{\mathrm{k}}_{2}}(\overrightarrow{\mathrm{q}})+S_{2 ; \overrightarrow{\mathrm{k}}_{1} \overrightarrow{\mathrm{k}}_{2}}(\overrightarrow{\mathrm{q}})\right] \Theta\left(\overrightarrow{\mathrm{k}}_{1}, \overrightarrow{\mathrm{q}}\right) \Theta\left(-\overrightarrow{\mathrm{k}}_{2}, \overrightarrow{\mathrm{q}}\right) \\
& \equiv \int_{0}^{\infty} d q \epsilon_{c}(q)
\end{aligned}
$$

we obtain

$$
\begin{aligned}
\left.\left.\left.\epsilon_{c}=\left\lfloor\frac{1}{2 N}\right]\left[\frac{1}{\alpha r_{s}}\right] \int \frac{2}{\pi^{2}}\right] \int d \overrightarrow{\mathrm{q}} \frac{1}{q^{2}} \sum_{\overrightarrow{\mathrm{k}}_{1} \overrightarrow{\mathrm{k}}_{2}}\left[2 S_{2 ; \overrightarrow{\mathrm{k}}_{1} \overrightarrow{\mathrm{k}}_{2}}(\overrightarrow{\mathrm{q}})+\left(\alpha r_{s}\right)\right] \frac{4}{3 \pi N}\right] \int \frac{1}{e}\right]\left[\frac{1}{q_{\mathrm{ex}}^{2}}\right]\left[1+2 f_{\overrightarrow{\mathrm{k}}_{1}}^{R}\left(\overrightarrow{\mathrm{q}}_{\mathrm{ex}}\right)\right] \\
\times\left[1+2 f_{-\overrightarrow{\mathrm{k}}_{2}}^{R}\left(\overrightarrow{\mathrm{q}}_{\mathrm{ex}}\right)\right] \Theta\left(\overrightarrow{\mathrm{k}}_{1}, \overrightarrow{\mathrm{q}}\right) \Theta\left(-\overrightarrow{\mathrm{k}}_{2}, \overrightarrow{\mathrm{q}}\right)
\end{aligned}
$$

where the superscript $R$ indicates the exact RPA quantity is to be used. A simple shift in the origin of the $q$ integration in the second term of Eq. (3.10) enables us to write it as 


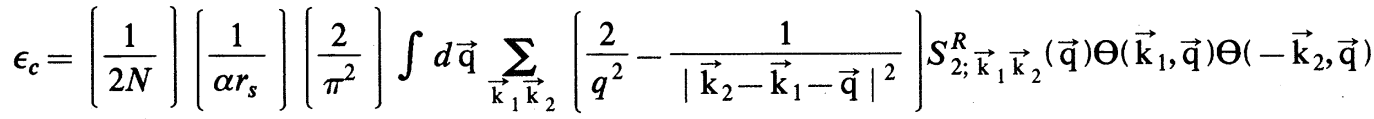

$$
\begin{aligned}
& \equiv \epsilon_{c}^{\mathrm{RPA}}+\epsilon_{c}^{\mathrm{RPAEX}(1)},
\end{aligned}
$$

which is precisely the expression used by Freeman. ${ }^{13}$ In so far as this approximation expressed by Eqs. (3.10) and (3.11) contains all ring diagrams plus those obtained from them by making one and only one exchange, it is a consistent (manifestly antisymmetric) lowest-order approximation for the inclusion of exchange effects, and henceforth we refer to it as the RPA + RPAEX(1) approximation.

Before we discuss the further evaluation of the expression (3.11), it is important to realize the physical difference between the RPA + RPAEX and RPA + RPAEX(1) approximations, and for this purpose it is most illustrative to consider the respective contributions to the correlation energy from large momentum transfers, $q \gg k_{1}, k_{2}$. It is seen from Eq. (3.11) that in the latter version these contributions are just half that in RPA. This clearly arises because the contribution from parallel-spin pairs is just canceled by its exchange, whereas the contribution from antiparallel-spin pairs remain unaltered. Actually solving the full RPA + RPAEX equations (3.2) - (3.4), the parallel-spin pair contribution still vanishes for high $q$ as is seen from Eq. (3.3), but the antiparallel-spin pair contribution clearly also changes, and it is this which makes the difference between the two. The reason that the antiparallel part of $S_{2}$ changes in RPA + RPAEX approximation is because the functions $f$ on the right-hand side of Eq. (3.2) are the sums of parallel and antiparallel parts [see Eqs. (3.4) and (3.5)], and in this case the parallel contribution now approaches zero as opposed to the RPA where both are equal. Iterating once, to obtain RPA + RPAEX(1) values, instead of solving the full RPA + RPAEX equations self-consistently, therefore corresponds to neglecting the influence on the screening of antiparallel-spin pairs of the disappearance of the (high-momentum transfer) correlated parallel-spin pairs. By solving the equations (3.2)-(3.5) selfconsistently, an additional screening mechanism is thus introduced which lowers the correlation energy (i.e., makes it more negative) or decreases the (positive) exchange energy. We note that by writing Eqs. (3.2) - (3.5) in their original dimensional units and by taking the limit of large $q$, it can readily be seen that the total $S_{2}$ [defined in Eq. (3.5)] just equals the same $S_{2}$ in RPA but at half the density. Opposed to that, the RPA + RPAEX(1) approximation yields an RPA value of $S_{2}$ at even lower density. Since exchange can hardly do more than cause the absence of correlated parallel-spin pairs, it is clear that iterating the RPA + RPAEX equations just once about the RPA solution certainly must overestimate the effects of exchange.

In order to compare our results with those of Freeman, ${ }^{13}$ and in order to test our approximation schemes later, we have evaluated the exact expression (3.11) for the correlation energy in the approximation RPA + RPAEX(1), using the exact RPA results for $S_{2}$ from I. Making the simple change of variables indicated in Eq. (3.20) of I, Eq. (3.11) here may be written as

$$
\begin{aligned}
\epsilon_{c}=-\frac{3}{16 \pi^{5}} \int_{\widetilde{\Gamma}} \frac{d \overrightarrow{\mathrm{q}}}{q^{2}} \int_{\widetilde{\Gamma}} d \vec{\kappa}_{1} \int_{\widetilde{\Gamma}} d \vec{\kappa}_{2} \frac{\widetilde{g}_{R}\left(\vec{\kappa}_{1}\right) \widetilde{g}_{R}\left(\vec{\kappa}_{2}\right)}{\overrightarrow{\mathrm{q}} \cdot\left(\vec{\kappa}_{1}+\vec{\kappa}_{2}\right)} \\
\quad \times\left(\frac{2}{q^{2}}-\frac{1}{\left|\vec{\kappa}_{1}+\vec{\kappa}_{2}\right|^{2}}\right) \\
\equiv \epsilon_{c}^{\mathrm{RPA}}+\epsilon_{c}^{\mathrm{RPAEX}(1)},
\end{aligned}
$$

in the notation of Eqs. (3.16) and (3.23) of I. Using Eqs. (3.26) and (3.19) of I, the result for the (direct) RPA contribution $\epsilon_{c}^{\mathrm{RPA}}$ is readily shown to be identical to the final result (3.61) of I. On the other hand, the (exchange) term $\epsilon_{c}^{\mathrm{RPAEX}(1)}$ in this approximation is evaluated numerically after further analysis which we relegate to Appendix A. It is seen there that use of an appropriate coordinate system (cylindrical polars) reduces the ninefold integral involved in the (second term of) Eq. (3.12) to a five-dimensional integration which we perform numerically. The values for the g.s. energy that we find in this approximation are in excellent agreement with those of Freeman ${ }^{13}$; and these values are quoted later in Table I.

We have made no "exact" numerical calculations beyond this "simple" RPA + RPAEX(1) approxi- 


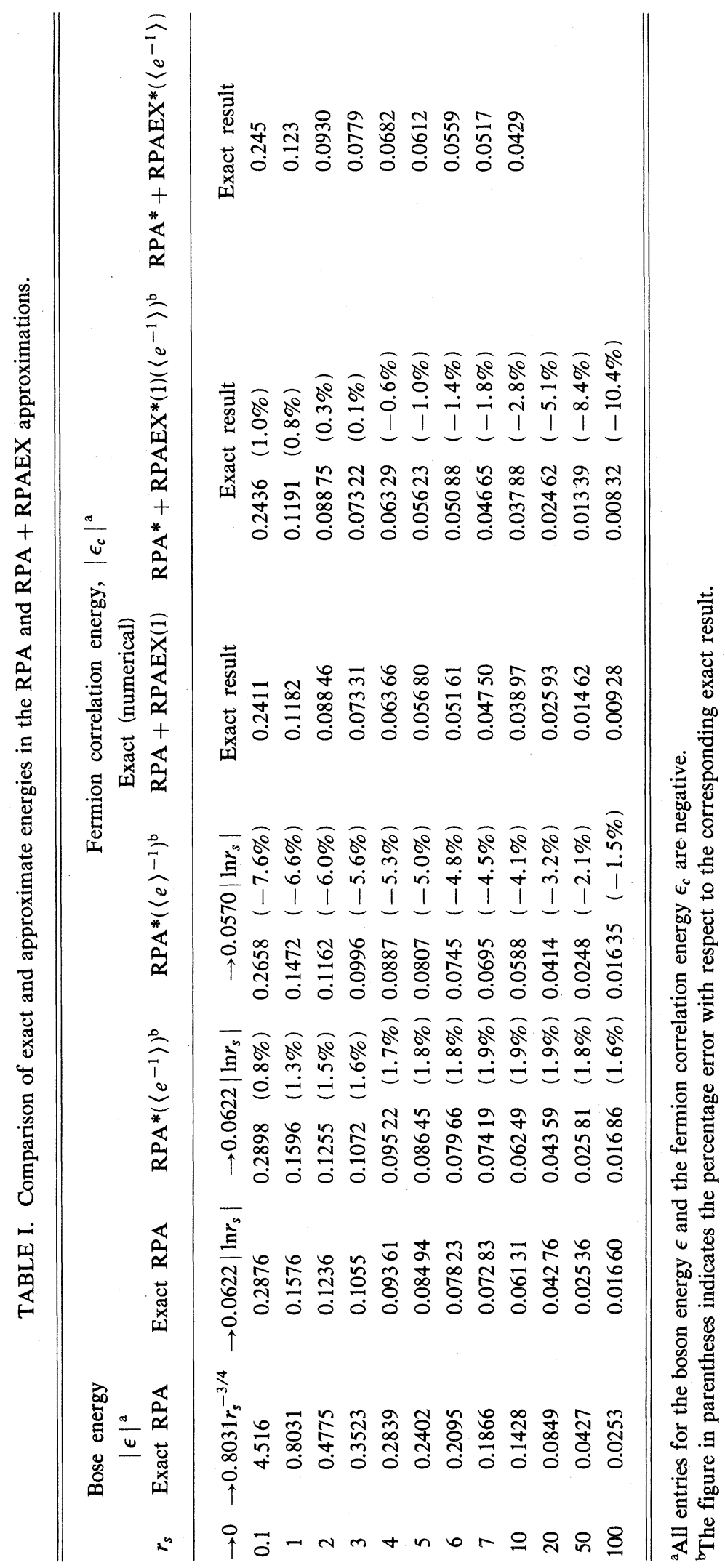


mation to the complete SUB2 scheme. Although further calculations would not seem to be wholly outside the scope of present computers, it is our intent now, however, to develop further approximations to enable us more readily to solve the full SUB2 (and beyond), as already discussed above. In the remainder of this section we will derive in detail these approximations (motivated by the much simpler analogous Bose results) for the RPA and RPA + RPAEX equations, and check their accuracy by comparing with the known exact RPA and RPA + RPAEX(1) results. Armed with the excellent agreement that we find, as described below, we thus proceed with confidence to include systematically more and more terms of the complete SUB2 in the later sections.

As mentioned earlier in this section, the motivation is to reduce the complexity of the fermion equations to basically no more than that of the corresponding boson equations treated in Sec. II by state-averaging away the effects of Fermi statistics. Keeping in mind, however, the important restriction induced by the exclusion principle, we are led to our basic approximation:

$$
\begin{aligned}
& S_{2 ; \overrightarrow{\mathrm{k}}_{1} \overrightarrow{\mathrm{k}}_{2}}^{\sigma_{1} \sigma_{2}}(\overrightarrow{\mathrm{q}}) \rightarrow\left\langle S_{2}^{\sigma_{1} \sigma_{2}}(q)\right\rangle \Theta\left(\overrightarrow{\mathrm{k}}_{1}, \overrightarrow{\mathrm{q}}\right) \Theta\left(-\overrightarrow{\mathrm{k}}_{2}, \overrightarrow{\mathrm{q}}\right), \\
& S_{2 ; \overrightarrow{\mathrm{k}}_{1} \overrightarrow{\mathrm{k}}_{2}}(\overrightarrow{\mathrm{q}}) \rightarrow\left\langle S_{2}(q)\right\rangle \Theta\left(\overrightarrow{\mathrm{k}}_{1}, \overrightarrow{\mathrm{q}}\right) \Theta\left(-\overrightarrow{\mathrm{k}}_{2}, \overrightarrow{\mathrm{q}}\right) .
\end{aligned}
$$

With these replacements, the correlation energy of Eq. (3.9) becomes

$$
\epsilon_{c} \rightarrow\left(\frac{1}{\alpha r_{s}}\right)\left(\frac{2}{\pi}\right) \int_{0}^{\infty} d q \bar{S}_{2}(q),
$$

with the definitions,

$$
\begin{aligned}
& {\overline{S_{2}}}^{\sigma_{1} \sigma_{2}}(q) \equiv P^{2}(q) N\left\langle S_{2}^{\sigma_{1} \sigma_{2}}(q)\right\rangle, \\
& \bar{S}_{2}(q) \equiv P^{2}(q) N\left\langle S_{2}(q)\right\rangle,
\end{aligned}
$$

and in terms of the function $P(q)$ defined by

$$
\begin{aligned}
P(q) & \equiv \frac{2}{N} \sum_{\overrightarrow{\mathrm{k}}} \Theta(\overrightarrow{\mathrm{k}}, \overrightarrow{\mathrm{q}}) \\
& =\left\{\begin{array}{l}
\frac{3}{4} q-\frac{1}{16} q^{3}, \quad q \leq 2 \\
1, q>2
\end{array}\right.
\end{aligned}
$$

and where the last result is valid in the thermodynamic limit. A comparison of the energy expression (3.14) with the corresponding Bose expression (2.18) shows that $\bar{S}_{2}(q)$ in the fermion case is the exact counterpart of the boson $S_{2}(q)$. Both functions are proportional to corresponding functions $\epsilon(q)$, which are defined in Eqs. (2.18) and (3.9) and which measure the contributions to the g.s. energy from different values of the momentum transfer. These two functions are compared at $r_{s}=1$ in RPA in Fig. 3. It is seen that the exact fermion RPA curve (from I) is similar to the exact boson RPA curve (from Sec. II) for large $q$ but that for small $q$ the curves completely differ. This latter difference is so dramatic that one may naively imagine that a useful approximation cannot arise from these ideas. Fortunately this is simply not so, as we now explain.

Making the replacement (3.13) into the exact fermion RPA equations [obtained from Eqs. (3.2) - (3.4) by neglecting the second term on the right-hand side of Eq. (3.3)], and summing over the filled Fermi sea for $\overrightarrow{\mathrm{k}}_{1}$ and $\overrightarrow{\mathrm{k}}_{2}$, we obtain

$$
\begin{aligned}
{\overline{S_{2}}}_{2}(q)= & -\left(\alpha r_{s}\right)\left(\frac{4}{3 \pi}\right) \frac{1}{q^{2}}\left\langle e^{-1}\right\rangle P^{2}(q) \\
& \times\left[1+\bar{S}_{2}(q) / P(q)\right]^{2},
\end{aligned}
$$

as the state-averaged Fermi RPA equation, where the average $\langle X\rangle=\langle X(q)\rangle$ of an arbitrary function $X\left(\overrightarrow{\mathrm{k}}_{1}, \overrightarrow{\mathrm{k}}_{2} ; \overrightarrow{\mathrm{q}}\right)$ has been defined as

$$
\begin{aligned}
\langle X\rangle \equiv & {\left[\sum_{\overrightarrow{\mathrm{k}}} \Theta(\overrightarrow{\mathrm{k}}, \overrightarrow{\mathrm{q}})\right]^{-2} } \\
& \times \sum_{\overrightarrow{\mathrm{k}}_{1} \overrightarrow{\mathrm{k}}_{2}} X\left(\overrightarrow{\mathrm{k}}_{1}, \overrightarrow{\mathrm{k}}_{2} ; \overrightarrow{\mathrm{q}}\right) \Theta\left(\overrightarrow{\mathrm{k}}_{1}, \overrightarrow{\mathrm{q}}\right) \Theta\left(-\overrightarrow{\mathrm{k}}_{2}, \overrightarrow{\mathrm{q}}\right) .
\end{aligned}
$$

The solution of Eq. (3.17) which remains bounded at large $q$ is

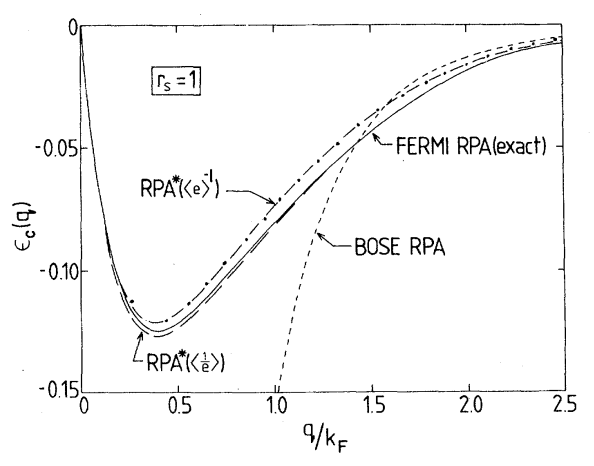

FIG. 3. Function $\epsilon(q)$ for bosons in RPA, and the function $\epsilon_{c}(q)$ for fermions in exact RPA and our two state-averaged approximations to it, for $r_{s}=1$. 


$$
\begin{aligned}
\bar{S}_{2}(q)= & -P(q)-\left[\frac{3 \pi}{8 \alpha r_{s}}\right] \frac{q^{2}}{\left\langle e^{-1}\right\rangle} \\
& +\left\{\left[\frac{3 \pi}{4 \alpha r_{s}}\right] \frac{q^{2} P(q)}{\left\langle e^{-1}\right\rangle}\right. \\
& \left.\left.+\left[\int \frac{3 \pi}{8 \alpha r_{s}}\right] \frac{q^{2}}{\left\langle e^{-1}\right\rangle}\right]^{2}\right\}^{1 / 2} .
\end{aligned}
$$

As already pointed out there is a certain remaining arbitrariness in our approximation, since we might equally multiply both sides of our original exact equation by an arbitrary function $f\left(\overrightarrow{\mathrm{k}}_{1}, \overrightarrow{\mathrm{k}}_{2} ; \overrightarrow{\mathrm{q}}\right)$ before performing the average. In particular, another possibility would be to multiply both sides first by $e$, and in the case of Eqs. (3.17) and (3.19) the quantity $\left\langle e^{-1}\right\rangle$ would have to be replaced by $\langle e\rangle^{-1}$. Because there are no a priori suitably compelling reasons to prefer one of these schemes over the other (although there would seem to be an intuitive preference for the former) we investigate them both further. The two approximations are henceforth referred to as $\operatorname{RPA}^{*}\left(\left\langle e^{-1}\right\rangle\right)$ and RPA* $\left(\langle e\rangle^{-1}\right)$, respectively. Both average quantities $\left\langle e^{-1}\right\rangle$ and $\langle e\rangle$ are evaluated in Appendix B and are henceforth regarded as known functions.

It is straightforward to show that in the highdensity limit the fermion correlation energy in RPA* is given from Eqs. (3.14) and (3.19) by

$$
\epsilon_{c} \underset{r_{s} \rightarrow 0}{\rightarrow} A \ln r_{s}+O\left(r_{s}^{0}\right)
$$

where the value of the constant $A$ depends on whether we work in the $\left\langle e^{-1}\right\rangle$ or $\langle e\rangle^{-1}$ averaging scheme. In both cases the values can be found analytically and are given, respectively, by

$$
A\left(\left\langle e^{-1}\right\rangle\right)=2 \pi^{-2}(1-\ln 2) \approx 0.0622
$$

in the $\left\langle e^{-1}\right\rangle$ scheme, and by

$$
A\left(\langle e\rangle^{-1}\right)=\frac{9}{16 \pi^{2}} \approx 0.0570
$$

in the $\langle e\rangle^{-1}$ scheme. By comparison with I we note that the $\operatorname{RPA}^{*}\left(\left\langle e^{-1}\right\rangle\right)$ approximation is exact

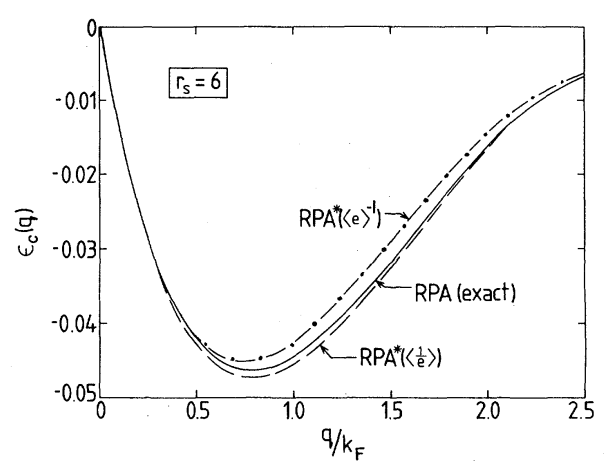

FIG. 4. As in Fig. 3 for the Fermion function $\epsilon_{c}(q)$, but for $r_{s}=6$.

in the high-density limit, whereas the $\operatorname{RPA}^{*}\left(\langle e\rangle^{-1}\right)$ approximation is in error by $8.3 \%$. Furthermore, from Table I we note that over the entire density range the $\left\langle e^{-1}\right\rangle$ averaging scheme is never in error by more than $2 \%$. Submitting our approximations to an even more stringent test we compare the contributions to $\epsilon_{c}$ from various momentum transfers by comparing the exact $\epsilon_{c}(q)$ from Eq. (3.9) evaluated with the exact RPA $S_{2}$ with its approximate counterpart from Eqs. (3.14) and (3.19). The results are shown for values $r_{s}=1$ and 6 in Figs. 3 and 4, respectively. It is seen that the relative errors in $\epsilon_{c}$ shown in Table I involve no cancellations at all from different $q$ regions, and we feel very justified in claiming that both approximation schemes are excellent, particularly the $\left\langle e^{-1}\right\rangle$ scheme. We remark finally that we can, of course, also write down analogous approximations for the TDA equations (which were also solved exactly in I). We find comparable agreement for TDA for both averaging schemes with exact results as discussed above for RPA.

From the overall excellent agreement so far obtained for our fermion approximation schemes, we feel justified in extending it to the full SUB2 approximation. In the remainder of this section we apply our fermion approximation (3.13) as a first step to the set of RPA + RPAEX equations (3.2) and (3.3) to obtain:

$$
\begin{aligned}
& \bar{S}_{2}^{\dagger \downarrow}(q)=-\left(\alpha r_{s}\right)\left(\frac{4}{3 \pi}\right) P^{2}(q) \frac{1}{q^{2}}\left\langle e^{-1}\right\rangle\left[1+\bar{S}_{2}(q) / P(q)\right]^{2}, \\
& \bar{S}_{2}^{\dagger \uparrow}(q)=-\left(\alpha r_{s}\right)\left(\frac{4}{3 \pi}\right) P^{2}(q)\left\{q^{-2}\left\langle e^{-1}\right\rangle\left[1+\bar{S}_{2}(q) / P(q)\right]^{2}-\left\langle q_{\mathrm{ex}}^{-2} e^{-1}\left[1+\bar{S}_{2}\left(q_{\mathrm{ex}}\right) / P\left(q_{\mathrm{ex}}\right)\right]^{2}\right\rangle\right\} .
\end{aligned}
$$


We note that in Eq. (3.24), a naive application of our basic approximation (3.13) leads to a supposedly state-averaged quantity $\bar{S}_{2}\left(q_{\mathrm{ex}}\right)$ which, however, still depends on the hole-state momenta through Eq. (3.8). It would seem consistent with our overall motivation to perform the average in Eq. (3.24) after the quantity $\bar{S}_{2}\left(q_{\mathrm{ex}}\right)$ has itself been fully averaged. Again, consistent with the underlying analogy with Bose systems, we make the simplest possible choice and replace $\overrightarrow{\mathrm{q}}_{\mathrm{ex}} \equiv \overrightarrow{\mathrm{k}}_{2}-\overrightarrow{\mathrm{k}}_{1}-\overrightarrow{\mathrm{q}}$ by $\overrightarrow{\mathrm{q}}$ in the argument of the function $\bar{S}_{2}(q)$. We test this further approximation below by comparing the resulting $\mathrm{RPA}^{*}+\mathrm{RPAEX}^{*}(1)$ approximation with the exact numerical results, and we find it to be excellent. After making this further approximation in Eq. (3.24), Eqs. (3.23) and (3.24) may be added to yield our RPA* + RPAEX* approximation:

$$
\begin{aligned}
\bar{S}_{2}(q)= & -\left(\alpha r_{s}\right)\left(\frac{4}{3 \pi}\right) P^{2}(q) v(q)\left\langle e^{-1}\right\rangle \\
& \times\left[1+\bar{S}_{2}(q) / P(q)\right]^{2},
\end{aligned}
$$

with

$$
v(q) \equiv q^{-2}-\frac{1}{2}\left\langle q_{\mathrm{ex}}^{-2} e^{-1}\right\rangle\left\langle e^{-1}\right\rangle^{-1} .
$$

Evaluation of the function $\left\langle q_{\mathrm{ex}}^{-2} e^{-1}\right\rangle$ is nontrivial and is discussed in Appendix C. We remark only that it must ultimately be evaluated numerically, and that its evaluation must be handled with great care (for reasons discussed below). Henceforth, we regard it as a known function.

It is interesting to note that the first-order iterate of Eq. (3.25) in powers of the potential (or equivalently about the zeroth-order solution $S_{2}=0$ appropriate to the high-density limit $r_{s} \rightarrow 0$ so long as the terms under discussion do not diverge) leads to a first-order exchange contribution $\bar{S}_{2 ; \text { ex }}^{(1)}$ to $\bar{S}_{2}$ of

$$
\bar{S}_{2 ; \mathrm{ex}}^{(1)}(q)=\left(\alpha r_{s}\right)\left[\frac{2}{3 \pi}\right] P^{2}(q)\left\langle q_{\mathrm{ex}}^{-2} e^{-1}\right\rangle
$$

and hence to a second-order (in powers of the potential) exchange energy $\epsilon_{2}^{(b)}$ given by inserting Eq. (3.27) into Eq. (3.14). Using definitions (3.18), (3.7), and (3.8), we readily find that $\epsilon_{2}^{(b)}$ may be written as

$$
\epsilon_{2}^{(b)}=\frac{3}{16 \pi^{5}} \int \frac{d \overrightarrow{\mathrm{q}}}{q^{2}} \int d \overrightarrow{\mathrm{k}}_{1} \int d \overrightarrow{\mathrm{k}}_{2} \frac{1}{\left|\overrightarrow{\mathrm{k}}_{2}-\overrightarrow{\mathrm{k}}_{1}-\overrightarrow{\mathrm{q}}\right|^{2}} \frac{1}{\overrightarrow{\mathrm{q}} \cdot\left(\overrightarrow{\mathrm{k}}_{1}-\overrightarrow{\mathrm{k}}_{2}+\overrightarrow{\mathrm{q}}\right)} \Theta\left(\overrightarrow{\mathrm{k}}_{1}, \overrightarrow{\mathrm{q}}\right) \Theta\left(-\overrightarrow{\mathrm{k}}_{2}, \overrightarrow{\mathrm{q}}\right),
$$

which is the exact result given by Gell-Mann and Brueckner, ${ }^{11}$ and evaluated analytically by Onsager, Mittag, and Stephen ${ }^{14}$ as

$$
\begin{aligned}
\epsilon_{2}^{(b)} & =\frac{1}{3} \ln 2-\frac{3}{2 \pi^{2}} \zeta(3) \\
& \approx 0.04836 .
\end{aligned}
$$

As a check on our numerical integration of the function $\left\langle q_{\mathrm{ex}}^{-2} e^{-1}\right\rangle$ from Eqs. (C3) we have evaluated $\epsilon_{2}^{(b)}$ numerically by inserting Eq. (3.27) into Eq. (3.14) and performing the integral by numerical quadrature. By comparison with the exact result (3.29) we find a value $\epsilon_{2}^{(b)} \approx 0.04834$.

Before we proceed with a fuller discussion of the RPA* + RPAEX* equations (3.23) - (3.25), we point out that the exchange contributions to the g.s. energy are extremely sensitive to any approximations made in their evaluation. We have already seen that our approximation is exact for the second-order exchange energy $\epsilon_{2}^{(b)}$. By contrast, in an earlier discussion of the exchange corrections to the RPA, Hubbard, ${ }^{15}$ working in a Green's- function (dielectric) formalism, proposed the approximation of replacing $q_{\mathrm{ex}}^{-2}$ by a state-averaged value of $\left(q^{2}+1\right)^{-1}$, whereas in our scheme the analogous replacement is clearly $q_{\mathrm{ex}}^{-2} \rightarrow\left\langle q_{\mathrm{ex}}^{-2} e^{-1}\right\rangle /\left\langle e^{-1}\right\rangle$ as may be seen from Eq. (3.26). Since our approximation is exact for $\epsilon_{2}^{(b)}$, it is of some interest to compare the two expressions, which we do in Fig. 5. Although the Hubbard approximation leads to a value of $\epsilon_{2}^{(b)} \approx 0.036$ [c.f. the exact value (3.29)], it is seen that the error in the momentum distribution is even worse than this value might suggest. While the Hubbard approximation underestimates the contribution to the correlation energy from values of $q \geqslant 1$, it very seriously overestimates the contributions from very small values of $q$. The difference is most drastic at $q \rightarrow 0$, where it can be shown

$$
\frac{\left\langle q_{\mathrm{ex}}^{-2} e^{-1}\right\rangle}{\left\langle e^{-1}\right\rangle} \underset{q \rightarrow 0}{\rightarrow} \frac{3(2 \ln 2-1)}{8(1-\ln 2)} \approx 0.472,
$$

whereas the corresponding value in the Hubbard approximation is unity.

Before evaluating the g.s. energy arising from 


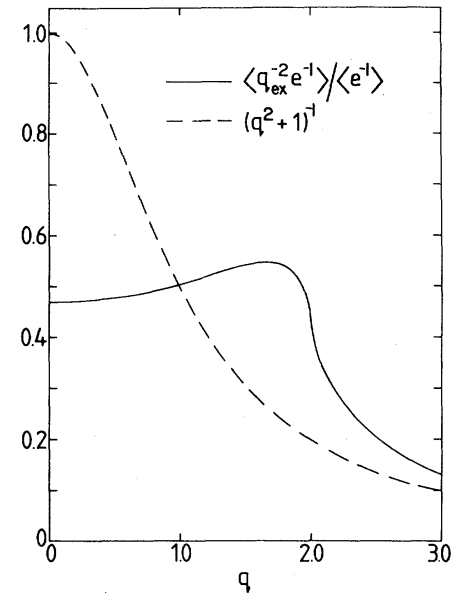

FIG. 5. Present state-averaged approximation $\left\langle q_{\mathrm{ex}}^{-2} e^{-1}\right\rangle /\left\langle e^{-1}\right\rangle$ to the exchange Coulomb potential $q_{\mathrm{ex}}^{-2}$ compared to the corresponding Hubbard approximation $\left(q^{2}+1\right)^{-1}$.

approximation (3.25) we may make one last check of our approximation scheme by comparing with the exact RPA + RPAEX(1) results of Eq. (3.12) discussed above. The analogous RPA*

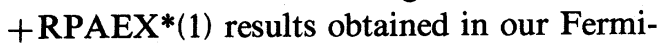
averaging scheme described above derive from iterating Eq. (3.25) once, but now about the RPA* solution $\bar{S}_{2}^{R}$ to Eq. (3.17) as the zeroth-order iterate. Inserting the resulting first-order iterate into Eq. (3.14) we obtain the correlation energy in the RPA* + RPAEX*(1) scheme as

$$
\begin{gathered}
\epsilon_{c}=-\frac{8}{3 \pi^{2}} \int_{0}^{\infty} d q P^{2}(q)\left\langle e^{-1}\right\rangle v(q) \\
\times\left[1+\bar{S}_{2}^{R}(q) / P(q)\right]^{2} \\
=\left[\frac{1}{\alpha r_{s}}\right]\left[\frac{2}{\pi}\right] \int_{0}^{\infty} d q q^{2} v(q) \bar{S}_{2}^{R}(q),
\end{gathered}
$$

where $\bar{S}_{2}^{R}(q)$ is given in Eq. (3.19). In Table I we compare the correlation energy from Eq. (3.31) [using the $\mathrm{RPA}^{*}\left(\left\langle e^{-1}\right\rangle\right)$ result for $\left.\bar{S}_{2}^{R}(q)\right]$ with the exact result from Eq. (3.12). It is seen that in the range of metallic densities the error induced by our fermion averaging procedure is very small. Thus at $r_{s}=4$, for example, the error is only $0.6 \%$ and in the metallic range $1<r_{s}<6$ it never exceeds $1.4 \%$. In this most interesting region, therefore, the error is even smaller than that of the RPA* $\left(\left\langle e^{-1}\right\rangle\right)$ scheme. Finally, we note that in order to gain some idea of the error involved in the full RPA* + RPAEX* scheme of Eq. (3.25), we might imagine iterating this equation further, around the RPA* solution. Since this doubtlessly leads to a rapidly converging result, it seems reasonable that the error involved in the full RPA* + RPAEX* solution should be of the same order as that obtained from Eq. (3.31) discussed above.

Finally, the correlation energies corresponding to the full RPA* $+\operatorname{RPAEX}^{*}\left(\left\langle e^{-1}\right\rangle\right)$ scheme of Eq. (3.25) are given in the last column of Table $I$, and in Fig. 6 we show the corresponding momentum distribution $\epsilon_{c}(q)$ in comparison to the same quantity in the RPA* $+\operatorname{RPAEX}^{*}(1)\left(\left\langle e^{-1}\right\rangle\right)$ and RPA* $\left(\left\langle e^{-1}\right\rangle\right)$ schemes, at a value $r_{s}=4$. From Table I we see that the absolute value of the correlation energy in the RPA* + RPAEX* scheme is about $8 \%$ greater than that of the RPA* + RPAEX*(1) scheme. As already pointed out, the RPA + RPAEX(1) approximation overestimates the effect of exchange, and from Fig. 6 we see that this overestimation occurs for relatively small momentum transfers only, in the range $0.3 \lesssim q \lesssim 1.6$ (in units of $k_{F}$ as usual).

We remark finally that the RPA + RPAEX curves in Fig. 6 have a remarkable form around $q=2$, which looks almost like a discontinuity. A similar behavior is also found for all values of $r_{s}$ that we have examined, viz., $0.1 \leq r_{s} \leq 10^{5}$. This behavior is certainly not an artifact of our fermion approximations since it occurs also for the exact solutions of the RPA + RPAEX(1) approximation (denoted by crosses in Fig. 6). To examine this behavior more closely we plot in Fig. 7, for $r_{s}=4$, in the $\mathrm{RPA}^{*}+\mathrm{RPAEX}^{*}\left(\left\langle e^{-1}\right\rangle\right)$, the solution $\bar{S}_{2}(q)$ to Eq. (3.25), together with the separate

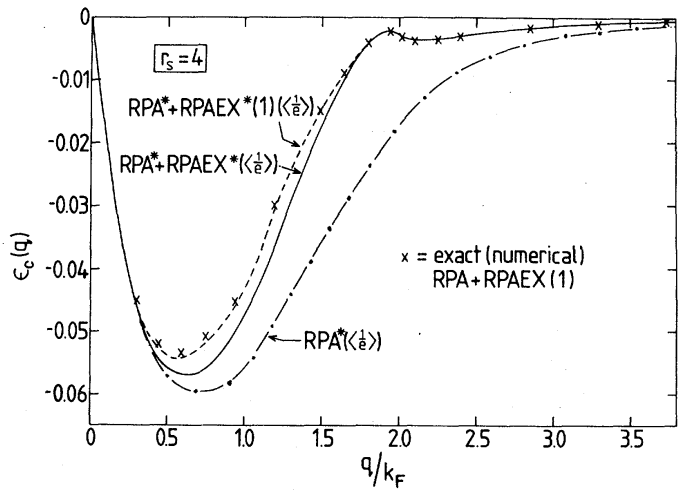

FIG. 6. Function $\epsilon_{c}(q)$ for $r_{s}=4$ in various approximations: $\operatorname{RPA}^{*}\left(\left\langle e^{-1}\right\rangle\right)$; $\operatorname{RPA}^{*}+\operatorname{RPAEX}^{*}(1)\left(\left\langle e^{-1}\right\rangle\right)$; RPA $^{*}+\operatorname{RPAEX}^{*}\left(\left\langle e^{-1}\right\rangle\right)$. The crosses denote exact (numerical) results in the RPA + RPAEX(1) approximation. 


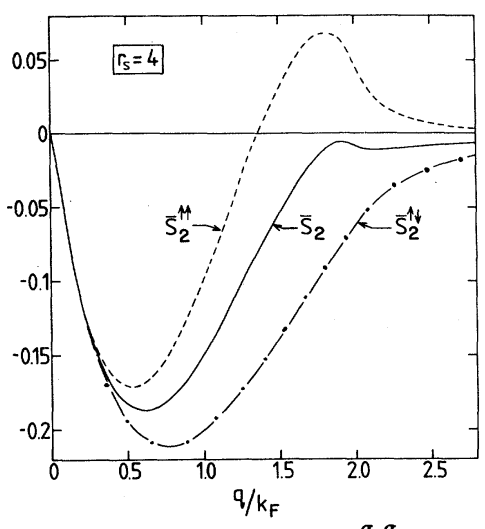

FIG. 7. State-averaged functions $\bar{S}_{2}^{\sigma_{1} \sigma_{2}}(q)$ in the $\operatorname{RPA}^{*}+\operatorname{RPAEX} *\left(\left\langle e^{-1}\right\rangle\right)$ approximation for parallel$(\uparrow \uparrow)$ and for antiparallel- $(\uparrow \downarrow)$ spin pairs, and for their average $\bar{S}_{2}(q)$ for $r_{s}=4$.

antiparallel- and parallel-spin contributions $\bar{S}_{2}^{\uparrow \downarrow}(q)$ and $\bar{S}_{2}^{\dagger \dagger}$ obtained from it by Eqs. (3.23)-(3.25) as

$$
\begin{aligned}
& \bar{S}_{2}^{\dagger \downarrow}(q)=\bar{S}_{2}(q) / q^{2} v(q), \\
& \bar{S}_{2}^{\dagger \uparrow}(q)=\bar{S}_{2}(q)\left[2-1 / q^{2} v(q)\right],
\end{aligned}
$$

and which are themselves proportional to the contributions of antiparallel- and parallel-spin pairs to $\epsilon_{c}(q)$ [c.f. Eqs. (3.5) and (3.9)]. We see that what appeared to be the peculiar behavior of $\epsilon_{c}(q)$ near $q=2$ is just due to the maximum of the parallel $\operatorname{spin} \bar{S}_{2}^{\dagger \dagger}$ at $q \approx 1.8$. Both phenomena are precisely related to the rapid decrease of the function $\left\langle q_{\mathrm{ex}}^{-2} e^{-1}\right\rangle$ around $q=2$ which can be seen from Fig. 5, and, in fact, the slope of this function can be shown to have a logarithmic singularity at $q=2$. This behavior is yet another indication that the "almost singular" expressions which arise due to the singular nature of the Coulomb potential have to be treated with considerable care, as already pointed out in connection with the Hubbard approximation. From all that we have said it is clear that our approximation scheme seems to handle this point extremely well.

In the following sections we turn our attention to enlarging the approximation developed in this section, first by incorporating the ladder terms in Sec. IV, and then in Sec. V to the full SUB2 approximation and beyond.

\section{INCLUSION OF LADDER TERMS}

It has been emphasized by many authors ${ }^{16-22}$ in the past that the inclusion of ladder diagrams within perturbation theory is essential for a proper treatment of the short-range correlations between electrons. This is hardly surprising, since for small distances between two electrons the Coulomb force is strong, and it is well known in, for example, the microscopic theory of nuclear matter ${ }^{23}$ or of closed-shell nuclei, ${ }^{4}$ that to deal with strong forces at small distances the closed summation of ladder diagrams is vital. In the electron gas the importance of the ladder diagrams is reflected in the observation that the radial distribution function can for some densities become negative for small values of the interparticle distance, if the ladder terms are not included. Accordingly we turn our attention in the present section to proceeding beyond the RPA + RPAEX scheme discussed in Sec. III by including the ladder terms. In I we distinguished between three contributions to the complete ladder (CLAD) summation [of Eq. (2.23) in I] in the SUB2 approximation, viz., the particle-particle ladders (PPLAD, or simply LAD henceforth), the hole-hole ladders (HHLAD), and the mixed ladders (MLAD). In this section we shall deal only with particle-particle ladders, and we denote by LAD the corresponding contribution to our equations.

The LAD term of Eqs. (2.23) and (2.15) of I is seen to arise in Eq. (2.10) of I from the first term on the right-hand side of this exact equation for $S_{2}$. If all other terms on the right-hand side are neglected, and furthermore the hole energies on the left-hand side are replaced by their kinetic parts only [viz., the first term only in Eq. (2.11) of I], we obtain the so-called Bethe-Goldstone equation:

$$
\begin{aligned}
& \left\langle\rho_{1} \rho_{2}\right|\left[T(1)+T(2)-\left\langle v_{1}|T| v_{1}\right\rangle\right. \\
& \left.-\left\langle v_{2}|T| v_{2}\right\rangle\right] S_{2}\left|v_{1} v_{2}\right\rangle_{A} \\
& =-\left\langle\rho_{1} \rho_{2}\left|V \Psi_{2}\right| v_{1} v_{2}\right\rangle_{A},
\end{aligned}
$$

where we have made the relevant simplifications for an infinite homogeneous system, by putting $S_{1}=0$, and considering plane-wave s.p. states. It is clear that the term in square brackets in Eq. (4.1) is just proportional to the quantity $e$ defined in Eq. (3.7). Inserting the operator identity

$$
\Psi_{2}=1+S_{2}
$$

appropriate for a homogeneous Fermi system [ $\mathrm{cf}$. Eq. (2.8) of I, with $S_{1}=0$ ] into the right-hand side of Eq. (4.1) and using plane-wave s.p. states, we readily find that (4.1) may be written in our by now familiar dimensionless variables as 


$$
\begin{aligned}
S_{2 ; \overrightarrow{\mathrm{k}}_{1} \overrightarrow{\mathrm{k}}_{2}}^{\sigma_{1} \sigma_{2}}(\overrightarrow{\mathrm{q}})= & -\left(\alpha r_{s}\right)\left(\frac{4}{3 \pi N}\right) \frac{1}{e}\left(\frac{1}{q^{2}}-\frac{\delta_{\sigma_{1} \sigma_{2}}}{q_{\mathrm{ex}}^{2}}\right) \\
& -\left(\alpha r_{s}\right)(4 \pi) \frac{1}{e} \int \frac{d \overrightarrow{\mathrm{q}}^{\prime}}{(2 \pi)^{3}} \frac{1}{\left|\overrightarrow{\mathrm{q}}-\overrightarrow{\mathrm{q}}^{\prime}\right|^{2}} S_{2 ; \overrightarrow{\mathrm{k}}_{1} \overrightarrow{\mathrm{k}}_{2}}^{\sigma_{1} \sigma_{2}}(\overrightarrow{\mathrm{q}}) \Theta\left(\overrightarrow{\mathrm{k}}_{1}, \overrightarrow{\mathrm{q}}^{\prime}\right) \Theta\left(-\overrightarrow{\mathrm{k}}_{2}, \overrightarrow{\mathrm{q}}^{\prime}\right) .
\end{aligned}
$$

Iterating Eq. (4.1) as it stands is readily seen to generate the particle-particle ladder diagrams. The situation is represented diagramatically in Fig. 8(a) for the antiparallel-spin pair only, for ease. (For the parallel-spin pair we get the extra driving term due to exchange antisymmetry.)

By taking other terms in the exact Eq. (2.10) of I for $S_{2}$ into account we may similarly regard them in an iteration procedure for the resulting equation as driving terms, generalizing the bare first-order (direct and exchange) potential term in Eq. (4.1b), and in this way the LAD term again builds up ladders between the two external particles [labeled $\rho_{1}$ and $\rho_{2}$ in Eq. (2.10) of I]. This procedure is illustrated in Fig. 8(b) where we include the RPA terms as well as the LAD term. We point out in passing that the RPA terms are generated in Eq. (2.10) of I from the first term in square brackets in each of the second and third

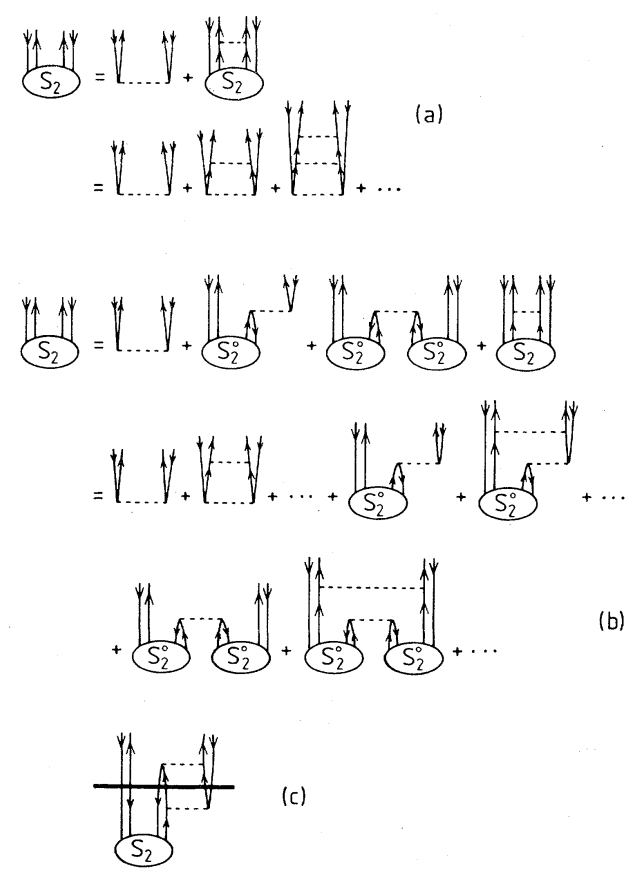

FIG. 8. (a) A schematic representation (for antiparallel-spin pairs) of the LAD summation, (b) a comparable schematic representation of the RPA + LAD summation, and (c) a particular diagram that does not arise in the previous iteration scheme. lines of the right-hand side, and from the first two terms in square brackets on the fourth line. Merely to remind ouselves of the iteration scheme we have in mind in Fig. 8(b), the role of the RPA terms as driving terms is indicated by labeling them $S_{2}^{0}$ in distinction to the variable $S_{2}$ in the LAD term on which we iterate; although otherwise, of course, $S_{2} \equiv S_{2}^{0}$ in this equation. Performing this iteration it is clear that, since the labels $\rho_{1}$ and $\rho_{2}$ appear in the first and second position in the bra state of each of the RPA terms, hence ladders can never be built up from the single rung corresponding to the potential $V(13), V(23)$, and $V(34)$ in these terms in Eq. (2.10) of I. Thus a diagram such as that in Fig. 8(c) can never arise from this iteration procedure, and the reader should have no trouble in convincing himself that any other iteration procedure of the RPA + LAD equation (gained by making another choice for which terms are first held constant as driving terms and which varied in the iteration) also does not lead to its inclusion. However, if as we have argued, the ladders generated by the LAD term are important, then we must expect that diagrams similar to that shown in Fig. 8(c), which also are contained in a class comprising comparable ladders, are also important. Furthermore, it is possible to show not only that the diagram of Fig. 8(c) is not generated in RPA + LAD approximation, but that it is contained nowhere in the SUB2 approximation to Eq. (2.10) of I, where $S_{3}$ and $S_{4}$ are set at zero. It is apparent that such terms are only included when $S_{3}$ and $S_{4}$ are themselves properly included. Crudely speaking, one effect of the proper inclusion of the three- and four-body terms $S_{3}$ and $S_{4}$ in the full Eq. (2.10) of I will be to replace the bare potentials $V(13), V(23)$, and $V(34)$ in the above-mentioned RPA terms by the "dressed" quantities $V(13) \Psi_{2}(13), V(23) \Psi_{2}(23)$, and $V(34) \Psi_{2}(34)$, respectively. However, an added complication which arises is that whilst in the SUB2 approximation only two-body energy denominators [c.f. Eq. (3.7)] appear, the inclusion of $S_{3}$ and $S_{4}$ leads to the appearance of real three- and four-body energy denominators. Thus, for example, the horizontal bar marked in Fig. 8(c) cuts 
three particle-hole pairs and leads in the coupledcluster formalism, as in the usual Goldstone formulation of perturbation theory in terms of timeordered diagrams, to a three-body energy denominator appearing in this term. We note that this is, in fact, sufficient reason for this diagram to be generated only in the three-body equation. (By contrast we also note that whereas other terms in Fig. 8(b) involve, for example, the simultaneous excitation of four or more particle-hole pairs, these arise from a SUB2 approximation, and as such are automatically included in the coupled-cluster framework in a "generalized time-ordering" (g.t.o.) sense for Goldstone perturbation theory. In turn this always leads to a factorization in terms of two-body energy denominators only. The interested reader is referred to Ref. 4 for a fuller discussion of this g.t.o. aspect of the coupled-cluster formalism, and how it leads to this so-called phenomenon of "upward factorization of the energy denominators"). Finally we note that it can rigorously be shown ${ }^{24}$ that the inclusion of $S_{3}$ and $S_{4}$ leads to partial diagram summations which justify the above replacements $V \rightarrow V \Psi_{2}$ in the RPA terms of SUB2 approximation, but only to the extent that two further conditions are fulfilled, viz., (i) the complications due to three- and four-body energy denominators can be neglected, and (ii) the function $S_{2}$ implicit in $\Psi_{2}$ [see Eq. (4.2)] is obtained by solving the Bethe-Goldstone equation (4.1). It cannot by contrast be shown by perturbation theory arguments that it is legitimate to compute the $S_{2}$ involved in the replacements $V \rightarrow V \Psi_{2}$ from the self-consistent solution to the full twobody approximation.

Summarizing our considerations so far, we have been led to two versions in which ladders can be introduced in our formalism. The first version is dictated precisely by the philosophy behind the SUB2 approximation (with $S_{3}$ and $S_{4}$ put to zero), and has the advantage of being completely systematic in the sense that it includes all particleparticle ladder terms which can be computed at a two-body level (involving no three- or four-body energy denominators). If ladders are important, however, then diagrams like that shown in Fig. 8(c) should also be important and it seems inconsistent to exclude them. The problem with our second version, described above and which does include them, is not with condition (i), which ought to be fulfilled at least to a first degree of approximation. Rather, it is condition (ii) which leads to a serious problem in introducing a subsidiary (Bethe-Goldstone) $S_{2}$.in which the very important RPA sceening effects are not included. Finally, therefore, to address this problem, we present below a heuristic argument which does not appeal to higher-order equations, for replacing this subsidiary Bethe-Goldstone $S_{2}$ by one which is the currently best available self-consistent solution of our full two-body equation. This final scheme we then denote as the third version for introducing ladders.

In order to motivate this third version, we compare the following Jastrow-type cluster expansion ansatz,

$$
\begin{aligned}
\Psi_{3} \rightarrow \Psi_{3}^{c}(123)= & {\left[1+S_{2}(12)\right]\left[1+S_{2}(23)\right] } \\
& \times\left[1+S_{2}(13)\right],
\end{aligned}
$$

with our coupled-cluster identity [c.f. Eq. (2.8c) of I],

$$
\begin{aligned}
\Psi_{3}(123)= & 1+S_{2}(12)+S_{2}(23) \\
& +S_{2}(13)+S_{3}(123),
\end{aligned}
$$

in the case that particle 2 is far from particles 1 and 3 in configuration $(\vec{r})$ space. The functions $S_{2}(12)$ and $S_{2}(23)$ are then both small and we have

$$
\Psi_{3}^{c} \approx\left[1+S_{2}(13)\right]\left[1+S_{2}(12)+S_{2}(23)\right]
$$

If we assume that $\Psi_{3}^{c}$ is correct in this limit, a comparison of Eqs. (4.4) and (4.5) leads in this limit to the relation,

$$
S_{3}(123) \approx S_{2}(13) S_{2}(12)+S_{2}(13) S_{2}(23) .
$$

We now use this result in the term $T_{2}$, for example, which represents the second line of the righthand side of Eq. (2.10) of I by first transforming the whole equation to configuration space, which just amounts to replacing the particle labels $\rho_{1}$ and $\rho_{2}$ by the configuration-space labels $\vec{r}_{1}$ and $\vec{r}_{2}$. This term then becomes

$$
T_{2} \equiv \sum_{v}\left\langle\overrightarrow{\mathrm{r}}_{1} \overrightarrow{\mathrm{r}}_{2} v\left|V(13)\left[S_{2}(23)+S_{2}(12)+S_{3}(123)\right]\right| v_{1} v_{2} v\right\rangle_{A},
$$

and we shall consider "proving" our result only in the limit that $\vec{r}_{1}$ and $\vec{r}_{2}$ are widely separated. In this case, since $V(13)$ is small unless particle 1 is close to particle 3 , the term in square brackets in Eq. (4.7) will 
have relevance only when particle 2 is far from both particles 1 and 3. We may then use the result (4.6) to show that in the limit that $\left|\vec{r}_{1}-\vec{r}_{2}\right| \rightarrow \infty$,

$$
T_{2} \rightarrow \sum_{v}\left\langle\overrightarrow{\mathrm{r}}_{1} \overrightarrow{\mathrm{r}}_{2} v\left|V(13) \Psi_{2}(13)\left[S_{2}(23)+S_{2}(12)\right]\right| v_{1} v_{2} v\right\rangle_{A}
$$

which is our desired result for this term. An analogous result clearly also holds for the term $T_{3}$, which represents the third line on the right-hand side of Eq. (2.10) of I, by the obvious symmetry between $T_{2}$ and $T_{3}$ under interchange of particles 1 and 2. Finally, an analogous consideration of $S_{4}$ in the limit of particles 1 and 2 far apart but with particles 3 and 4 close leads to the relation,

$$
\begin{aligned}
S_{4}(1234) \approx & S_{2}(34)\left[S_{2}(13) S_{2}(24)+S_{2}(23) S_{2}(14)+S_{3}(123)+S_{3}(124)\right] \\
& +\Psi_{2}(34)\left\{S_{2}(13) S_{2}(14)\left[1+S_{2}(24)+S_{2}(23)\right]+S_{2}(23) S_{2}(24)\left\{\left[1+S_{2}(14)+S_{2}(13)\right]\right\}\right. \\
& +\left\{S_{2}(34)\left[S_{2}(13)+S_{2}(14)+S_{2}(23)+S_{2}(24)\right]-S_{3}(134)-S_{3}(234)\right\}
\end{aligned}
$$

Whereas the first term in Eq. (4.9) gives precisely the result required for the term $T_{4}$ which represents the last two lines of Eq. (2.10) of I, namely in the limit $\left|\vec{r}_{1}-\vec{r}_{2}\right| \rightarrow \infty$,

$$
T_{4} \rightarrow \frac{1}{2} \sum_{v v^{\prime}}\left\langle\overrightarrow{\mathrm{r}}_{1} \overrightarrow{\mathrm{r}}_{2} v v^{\prime}\left|V(34) \Psi(34)\left[S_{2}(13) S_{2}(24)+S_{2}(23) S_{2}(14)+S_{3}(123)+S_{3}(124)\right]\right| v_{1} v_{2} v v^{\prime}\right\rangle_{A},
$$

the second and third terms in Eq. (4.9) gives an extra contribution which we ignore. Having thus motivated these results in the limit $\left|\vec{r}_{1}-\vec{r}_{2}\right| \rightarrow \infty$, we now define our third version of including ladders to be where in the RPA terms of Eq. (2.10) of I, the three operators $V$ in the second, third, and fourth lines of the righthand side are replaced (for all separations) by the respective operators $V \Psi_{2} \equiv G$, the so-called $G$ matrix. In this third version the resulting $G$ matrix is thus computed from the self-consistent solution $S_{2}$ to the full equation; whereas in the second version $G$ is replaced by $G_{\mathrm{BG}}$, computed from the solution to the BetheGoldstone (BG) equation (4.1).

In the remainder of this section we now derive explicit expressions for our three versions of including ladders in the state-averaging Fermi approximation of the preceding section. We start with the first version which is simply the SUB2 equation in the RPA + RPAEX + LAD approximation. According to Eqs. $(2.15)-(2.20)$ and (2.23) of $\mathrm{I}$, we have in this approximation

$$
\begin{aligned}
S_{2 ; \overrightarrow{\mathrm{k}}_{1} \overrightarrow{\mathrm{k}}_{2}}^{\sigma_{1} \sigma_{2}}(\overrightarrow{\mathrm{q}})= & -\left(\alpha r_{s}\right)\left(\frac{4}{3 \pi N}\right) \frac{1}{e} \int \frac{1}{q^{2}}\left[1+2 f_{\overrightarrow{\mathrm{k}}_{1}}(\overrightarrow{\mathrm{q}})\right]\left[1+2 f_{-\overrightarrow{\mathrm{k}}_{2}}(\overrightarrow{\mathrm{q}})\right] \\
& -\frac{\left.\delta_{\sigma_{1} \sigma_{2}}^{q_{\mathrm{ex}}^{2}}\left[1+2 f_{\overrightarrow{\mathrm{k}}_{1}}\left(\overrightarrow{\mathrm{q}}_{\mathrm{ex}}\right)\right]\left[1+2 f_{-\overrightarrow{\mathrm{k}}_{2}}\left(\overrightarrow{\mathrm{q}}_{\mathrm{ex}}\right)\right]\right)}{} \\
& -\left(\alpha r_{s}\right)(4 \pi)\left(\frac{1}{e}\right) \int \frac{d \overrightarrow{\mathrm{q}}^{\prime}}{(2 \pi)^{3}} \frac{1}{\left|\overrightarrow{\mathrm{q}}-\overrightarrow{\mathrm{q}}^{\prime}\right|^{2}} S_{2 ; \overrightarrow{\mathrm{k}}_{1} \overrightarrow{\mathrm{k}}_{2}}^{\sigma_{1} \sigma_{2}}\left(\overrightarrow{\mathrm{q}}^{\prime}\right) \Theta\left(\overrightarrow{\mathrm{k}}_{1}, \overrightarrow{\mathrm{q}}^{\prime}\right) \Theta\left(-\overrightarrow{\mathrm{k}}_{2}, \overrightarrow{\mathrm{q}}^{\prime}\right),
\end{aligned}
$$

where we have used the definitions (3.4) - (3.8).

In order to make further progress with Eq. (4.11) it seems almost inevitable that we are led to make the familiar approximation of replacing in the LAD term the $\Theta$ functions of Eq. (3.6) by their angle-averaged values,

$$
\Theta(\overrightarrow{\mathrm{k}}, \overrightarrow{\mathrm{q}}) \rightarrow \hat{\Theta}(k, q)=\Theta(1-k) \times\left\{\begin{array}{c}
0, q<1-k \\
{\left[\begin{array}{l}
\left.(k+q)^{2}-1\right] / 4 q k, \quad 1-k \leq q<1+k \\
1, q \geq 1+k
\end{array}\right.}
\end{array}\right.
$$


Applying now the fermion approximation already described to Eq. (4.11) and carrying out the angular integrations leads to

$$
\begin{aligned}
{\overrightarrow{S_{2}}}_{2}^{\sigma_{1} \sigma_{2}}(q)= & -\left(\alpha r_{s}\right)\left(\frac{4}{3 \pi}\right) P^{2}(q)\left\langle\frac{1}{e}\right\rangle\left[1+\bar{S}_{2}(q) / P(q)\right]^{2}\left[q^{-2}-\delta_{\sigma_{1} \sigma_{2}}\left\langle q_{\mathrm{ex}}^{-2} e^{-1}\right\rangle\left\langle e^{-1}\right\rangle^{-1}\right] \\
& -\frac{\alpha r_{s}}{\pi q}\left\langle\frac{1}{e}\right\rangle \int_{0}^{\infty} d q^{\prime} q^{\prime} \ln \left|\frac{q+q^{\prime}}{q-q^{\prime}}\right| \frac{P^{2}\left(q, q^{\prime}\right)}{P^{2}\left(q^{\prime}\right)} \bar{S}_{2}^{\sigma_{1} \sigma_{2}}\left(q^{\prime}\right)
\end{aligned}
$$

where the function $P\left(q, q^{\prime}\right)$ is defined by

$$
P\left(q, q^{\prime}\right) \equiv \frac{2}{N} \sum_{\overrightarrow{\mathrm{k}}} \hat{\Theta}(k, q) \hat{\Theta}\left(k, q^{\prime}\right) .
$$

The explicit evaluation of $P\left(q_{1}, q_{2}\right)$ is straightforward, although tedious, and we quote only the final result. Since it is clear from the definition (4.14) that $P\left(q_{1}, q_{2}\right)=P\left(q_{2}, q_{1}\right)$ it is sufficient to consider only the case $q_{1} \leq q_{2}$. We find

$$
P\left(q_{1}, q_{2}\right)=P\left(q_{1}\right), \quad 0 \leq q_{1} \leq q_{2}, \quad q_{2} \geq 2
$$

where $P(q)$ is given by Eq. (3.16), and

$$
\begin{aligned}
P\left(q_{1}, q_{2}\right)=\frac{3}{16 q_{1} q_{2}} & {\left[\frac{1}{5} A_{5}^{-}+\left(1-\frac{1}{2} q_{1}\right) A_{4}^{-}+\frac{1}{2} q_{2} A_{4}^{+}+\frac{1}{3}\left(q_{1}^{2}+q_{2}^{2}-6 q_{1}+4\right) A_{3}^{-}\right.} \\
& \left.+\frac{2}{3} q_{2}\left(3-q_{1}\right) A_{3}^{+}+\left(q_{1}^{2}+q_{2}^{2}-2 q_{1}\right) A_{2}^{-}+2 q_{2}\left(1-q_{1}\right) A_{2}^{+}\right], 0 \leq q_{1} \leq q_{2}<2
\end{aligned}
$$

with the functions $A_{n}^{ \pm}$defined by

$$
A_{n}^{ \pm} \equiv A_{n}^{ \pm}\left(q_{1}, q_{2}\right) \equiv q_{1}^{n} \pm\left(q_{1}+q_{2}-2\right)^{n} \Theta\left(q_{1}+q_{2}-2\right) .
$$

It is readily seen that without making the angle-averaging approximation of Eq. (4.12), it would have been very difficult to have brought the LAD term into readily computable form. Fortunately this approximation would not seem to introduce much further inaccuracy into our final equations. It has certainly been very well tested in its application to the Bethe-Goldstone equation (4.1) for nuclear matter, ${ }^{23}$ and there is no reason to expect it not to be as excellent an approximation here. The fermion approximation itself is also thought not to introduce a larger error for the LAD term than that found in Sec. III for the RPAEX terms, where we could explicitly compare with exact results. Henceforth, we refer to Eq. (4.13) as the first version of our $\mathrm{RPA}^{*}+\mathrm{RPAEX}^{*}+\mathrm{LAD}^{*}\left(\left\langle e^{-1}\right\rangle\right)$ approximation.

In order to write the explicit forms of our second and third versions to include ladders, by comparison of Eqs. (4.1), (4.11), and (4.13) we are led to define a $G$ matrix in our state-averaged scheme by

$$
\left.G^{\sigma_{1} \sigma_{2}}(q)=\left(\alpha r_{s}\right)\left[\frac{4}{3 \pi} P^{2}(q) \mid \frac{1}{q^{2}}-\delta_{\sigma_{1} \sigma_{2}} \frac{\left\langle q_{\mathrm{ex}}^{-2} e^{-1}\right\rangle}{\left\langle e^{-1}\right\rangle}\right]+\frac{1}{\pi q} \int_{0}^{\infty} d q^{\prime} q^{\prime} \ln \left|\frac{q+q^{\prime}}{q-q^{\prime}}\right| \frac{P^{2}\left(q, q^{\prime}\right)}{P^{2}\left(q^{\prime}\right)} \bar{S}_{2}^{\sigma_{1} \sigma_{2}}\left(q^{\prime}\right)\right] \text {. }
$$

The equation defining our second version is then given in terms of this $G$ matrix by

$\bar{S}_{2}^{\sigma_{1} \sigma_{2}}(q)=-\left\langle e^{-1\rangle}\left\{G^{\sigma_{1} \sigma_{2}}(q)+\left[G_{\mathrm{BG}}^{\dagger \downarrow}(q)-\delta_{\sigma_{1} \sigma_{2}} G_{\mathrm{BG}}^{\dagger \downarrow}\left(\bar{q}_{\mathrm{ex}}\right)\right]\left[2 \frac{\bar{S}_{2}(q)}{P(q)}+\left(\frac{\bar{S}_{2}(q)}{P(q)}\right)^{2}\right]\right\}\right.$,

where $G_{\mathrm{BG}}^{\sigma_{1} \sigma_{2}}(q)$ is the corresponding BetheGoldstone $G$ matrix, which by comparison of Eqs. $\left(4.1^{\prime}\right),(4.13)$, and (4.16) is seen to be the solution of the equation

$$
\bar{S}_{2}^{\sigma_{1} \sigma_{2}}(q)=-\left\langle e^{-1}\right\rangle G^{\sigma_{1} \sigma_{2}}(q),
$$

with the $G$ matrix still defined by Eq. (4.16). Similarly, the equation defining our third version 
of including ladders is identical to Eq. (4.16) but with the replacement $G_{\mathrm{BG}}^{\uparrow \downarrow} \rightarrow G^{\uparrow \downarrow}$, and in this case Eqs. (4.16) and (4.17) are solved self-consistently. Concerning Eq. (4.17) we mention that the antiparallel-spin Bethe-Goldstone $G$ matrix $G_{\mathrm{BG}}^{\dagger \downarrow}$ appears even in the equation for parallel-spin pairs $\left(\sigma_{1}=\sigma_{2}\right)$ since these $G$ matrices replace a direct potential vertex $V$ in the simpler RPA + RPAEX Eqs. (3.23) and (3.24). It is also clear from a comparison of Eqs. (3.24) and (4.17) that in the exchange term of Eq. (4.17) we have been forced to make the replacement

$$
\left\langle e^{-1} q_{\mathrm{ex}}^{-2}\right\rangle \rightarrow\left\langle e^{-1}\right\rangle\left\langle q_{\mathrm{ex}}^{-2}\right\rangle
$$

which is certainly not a very good approximation. In fact about a $5 \%$ error would be incurred in the correlation energy at a density $r_{s}=4$ if the same replacement were made in Eq. (4.16) in evaluating the first term on the right-hand side of Eq. (4.17). In order to keep the approximation as accurate as possible, we insist that at least first-order (in powers of the potential, or where $G_{\mathrm{BG}}$ is replaced by $V$ is treated exactly, and hence the exchangemomentum argument $\bar{q}_{\text {ex }}$ of the exchange $G$ matrix in Eq. (4.17) is chosen to be

$$
\bar{q}_{\mathrm{ex}}=\left(\left\langle e^{-1}\right\rangle /\left\langle e^{-1} q_{\mathrm{ex}}^{-2}\right\rangle\right)^{1 / 2} .
$$

Since no error is then incurred in the leading term, we are confident that any extra error incurred by the replacement (4.19) in the derivation of Eq. (4.17) is very small.

We note, finally, before presenting results obtained from Eqs. (4.13), (4.16), and (4.17) that the numerical solution of these equations is not entirely trivial, due to the logarithmic singularity in the integral kernel. We found it convenient to subtract (and add exactly elsewhere) from both integrals the quantity

$$
q \frac{P^{2}(q, q)}{P^{2}(q)} \bar{S}_{2}^{\sigma_{1} \sigma_{2}}(q) \int_{0}^{100} d q^{\prime} \ln \left|\frac{q+q^{\prime}}{q-q^{\prime}}\right|
$$

in order to regularize the integral terms, and where the upper limit $q=100$ was used in most of our numerical work. The momentum-variable meshes used were typically as follows: 129 points in the range $\left(10^{-8}, 2.4\right), 65$ points in the range $(2.4,6.0), 33$ points in the range $(6.0,12.0)$, and 33 points in the range $(12.0,100)$. In each of these subintervals Romberg integration was used. Where necessary for very accurate results, as reported in Sec. II from solving Eq. (2.34) in the limit $r_{s} \rightarrow \infty$, for example, we have also made an end-point correction to take care of the range $(100, \infty)$ using the ap- propriate asymptotic form for $\bar{S}_{2}(q)$.

We give results in Table II for the correlation energy in the metallic density range, using both our first and third versions of incorporating particleparticle ladders in this RPA $^{*}+$ RPAEX $^{*}+$ $\operatorname{LAD}^{*}\left(\left\langle e^{-1}\right\rangle\right)$ approximation, and results are shown at $r_{s}=4$ for the state-averaged quantities $\bar{S}_{2}^{\dagger \uparrow}(q)$ and $\bar{S}_{2}^{\dagger \downarrow}(q)$ in Figs. 9 and 10 for the first and third versions, respectively. We do not present results for the second version since we found that in this approximation $\bar{S}_{2}$ becomes unphysical by taking complex values at small momenta $q$ (e.g., for $r_{s}=1$ and 6 this occurs, respectively, in the ranges $0<q \leqq 0.1$ and $0<q \leqq 0.4)$. This unphysical. behavior clearly confirms our earlier belief that this version could be seriously in error since (lowmomentum RPA) screening of the additional ladder interactions is neglected.

A comparison of the entries of Table II with those for the RPA* $\operatorname{RPAEX}^{*}\left(\left\langle e^{-1}\right\rangle\right)$ approximation from the last column in Table I shows that the first (SUB2) version of incorporating particleparticle ladders gives a substantial reduction in the absolute value of the correlation energy, whereas this effect is somewhat less in our third (selfconsistent $G$-matrix) version. A more revealing comparison can be made from Figs. 7, 9, and 10. Whereas the curves for $\bar{S}_{2}^{\sigma_{1} \sigma_{2}}$ are virtually identical in the low-momentum regime for Figs. 7 and 9 only up to $q \approx 0.15$, the curves of Figs. 7 and 10 are virtually identical for $q \leqq 0.3$. Since RPA + RPAEX effects dominate in the long-range limit (and become exact as $q \rightarrow 0$ ) it is clear that the less sophisticated SUB2 version of ladders artificially obscures the form of $\bar{S}_{2}^{\sigma_{1} \sigma_{2}}$ for small $q$. The general form of the curves in Figs. 7, 9, and 10 shows this same statement to hold for intermediate values of $q$ as well, up to $q \approx 1.6$. For values $q \gtrsim 1.6$, the SUB2 and self-consistent $G$ matrix versions of ladders give nearly identical values of $\bar{S}_{2}^{\dagger \downarrow}$, and again both are expected to be essentially exact in this short-range limit.

We have seen that not only our second version of ladder incorporation has to be discarded, but the same is also true of our first (SUB2) version. The inclusion of ladders reduced $\bar{S}_{2}^{\uparrow \downarrow}$ in magnitude by about $30 \%$ at $q=1$ (for $r_{s}=4$ ), and this very large effect at what is still a relatively small value of $q$ is simply not treated adequately in the SUB2 version. It is clear that both the long-range difficulties (at small $q$ ) and the short-range difficulties (at large $q$ ) are very adequately dealt with (by RPA and any version of ladders, respectively) insofar as they are 
TABLE II. Comparison of correlation energies in the RPA* + RPAEX*

$+\operatorname{LAD}^{*}\left(\left\langle e^{-1}\right\rangle\right)$ approximation for different versions of including the ladder term.

\begin{tabular}{lcccccc}
\hline \hline Version & 1 & 2 & 3 & 4 & 5 & 6 \\
\hline SUB2 & 1 & & & $r_{s}$ & \\
Self-consistent $G$ matrix & -0.11132 & -0.0810 & -0.0646 & -0.0543 & -0.0471 & -0.0417 \\
\hline
\end{tabular}

independent of each other. However, the strong "interference" of both effects at intermediate momenta forces us to go beyond SUB2 approximation, to take at least some three- and four-body effects of $S_{3}$ and $S_{4}$, respectively, into account. We have motivated our self-consistent $G$-matrix approach (which henceforth we refer to as the LAD version, since the first and second versions are now discarded) by using a Jastrow-type cluster decomposition of $S_{3}$ and $S_{4}$ which, on the one hand, should be asymptotically exact in the long-range limit and which, on the other hand, provided us with the extra terms which obviously allow for a more complete treatment of the short-range effects.

Before proceeding in the next section to examine the other terms we have neglected so far in the exact equation for $S_{2}$, we finally mention that in this and following sections we present results only for values of $r_{s}$ up to 5 or 6 because our numerical procedures simply failed to find solutions for $\bar{S}_{2}$ for lower densities in these approximations. In practice we found that the iteration schemes employed to solve the equations [e.g., Eqs. (4.16) and (4.17)] no longer converged at these low densities. At present we simply do not know whether these (explicitly nonlinear) equations describing the ap-

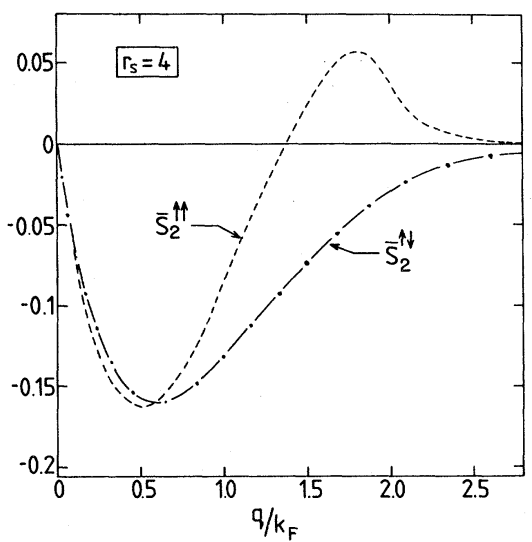

FIG. 9. As in Fig. 7, but in the RPA* + RPAEX* $+\operatorname{LAD}^{*}\left(\left\langle e^{-1}\right\rangle\right)$ approximation, and using the first (SUB2) version of incorporating ladders. proximations do not have (physically acceptable) solutions in this density range, or whether the problem lies with a need for a more sophisticated method of solution.

\section{STATE AVERAGING OF THE FULL SUB2 EQUATION AND FINAL RESULTS}

Until now we have studied the fermion SUB2 equations by taking into account only the RPA and RPAEX terms (in Sec. III), and later incorporating the LAD term (in Sec. IV) as well. We have argued that whereas the RPA term mainly determines the very important long-range (screening) behavior of the two-body cluster function $S_{2}$, the LAD term is responsible for the short-range behavior and indeed an accurate description of this limit even involves us in including some highercluster effects. Having studied these two limiting cases in some depth we intend now to consider all of the remaining terms of the SUB2 equations (2.15) of I that have so far been neglected. We remind the reader that these remaining terms are the following: (i) the Fock hole potential (FHP) and (ii) the (self-consistent) hole-potential (HP) terms which, in the absence of a Hartree holepotential term for the neutral Coulomb system

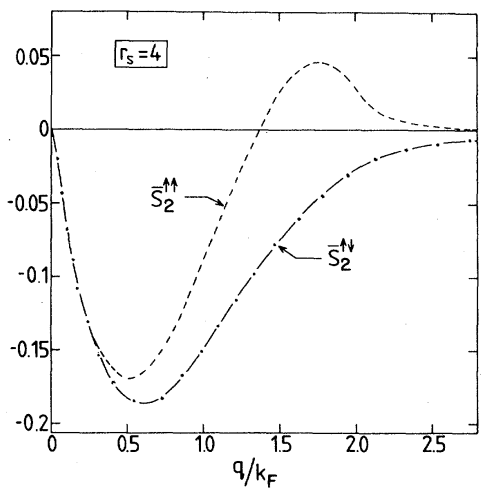

FIG. 10. As in Fig. 9, but using the third (selfconsistent $G$-matrix) version of incorporating ladders. 
considered here, together generate the fully selfconsistent complete hole-potential (CHP) insertions on the hole lines, (iii) a similar complete particlepotential (CPP) term which generates the fully self-consistent self-energy for particle states, (iv) a hole-hole ladder (HHLAD) term, and (v) a mixed ladder (MLAD) term which, together with the particle-particle ladder (LAD) term already considered, generate the complete ladder (CLAD) set of diagrams for two-particle and two-hole scattering within the many-body medium, (vi) the PHA and (vii) the PHB terms which generate two sorts of particle-hole ladder diagrams, and finally two extra exchange terms (viii) EE1 and (ix) EE2, which are required by Fermi statistics to preserve the overall antisymmetry of $S_{2}$.

We start by considering the PHB term, given by Eq. (2.25) of I. Using Eq. (3.13), and summing over the hole momenta $\overrightarrow{\mathrm{k}}_{1}$ and $\overrightarrow{\mathrm{k}}_{2}$ in accord with our state-averaging approximation, this term becomes

$$
\begin{aligned}
t_{\mathrm{PHB}} \equiv-2 \sum_{\overrightarrow{\mathrm{k}}_{1} \overrightarrow{\mathrm{k}}_{2}} \sum_{\overrightarrow{\mathrm{k}}} & V\left(\overrightarrow{\mathrm{k}}_{2}-\overrightarrow{\mathrm{k}}\right)\left\langle S_{2}^{s_{1} s_{2}}\left(\left|\overrightarrow{\mathrm{k}}+\overrightarrow{\mathrm{q}}-\overrightarrow{\mathrm{k}}_{2}\right|\right)\right\rangle \Theta\left(\overrightarrow{\mathrm{k}}_{1}, \overrightarrow{\mathrm{k}}+\overrightarrow{\mathrm{q}}-\overrightarrow{\mathrm{k}}_{2}\right) \\
& \times \Theta\left(\overrightarrow{\mathrm{k}}_{1}, \overrightarrow{\mathrm{q}}\right) \Theta\left(-\overrightarrow{\mathrm{k}}_{2}, \overrightarrow{\mathrm{q}}\right) \Theta\left(k_{F}-k\right)
\end{aligned}
$$

The problem that remains is how to deal with the state-averaged $S_{2}$ occurring in Eq. (5.1), since there are clearly many ways of performing this average. In a very similar situation in Sec. III, in connection with the RPAEX term [and see Eq. (3.24)], we found it a very successful approximation simply to average the vector argument of the function $S_{2}$. The success of this approximation seems to be linked to the fact that the summation (hole-momentum) variables are thereby replaced by just those values where the two-body potential $V$ occurring in the expression becomes largest, and hence most important. The situation in Eq. (5.1) is seen to be very similar, in that replacing the vector $\left(\vec{k}+\vec{q}-\vec{k}_{2}\right)$ by its hole-state average value $\vec{q}$, yields just the same result as by substituting in it the value $\overrightarrow{\mathrm{k}}_{2}=\overrightarrow{\mathrm{k}}$ where the potential $V\left(\overrightarrow{\mathrm{k}}_{2}-\overrightarrow{\mathrm{k}}\right)$ in Eq. (5.1) becomes singular. Performing this average yields the simple approximation,

$$
t_{\mathrm{PHB}} \approx-2 \sum_{\overrightarrow{\mathrm{k}}_{1} \overrightarrow{\mathrm{k}}_{2}} \sum_{\overrightarrow{\mathrm{k}}}^{k_{F}} V\left(\overrightarrow{\mathrm{k}}_{2}-\overrightarrow{\mathrm{k}}\right)\left\langle S_{2}^{s_{1} s_{2}}(q)\right\rangle \Theta\left(\overrightarrow{\mathrm{k}}_{1}, \overrightarrow{\mathrm{q}}\right) \Theta\left(-\overrightarrow{\mathrm{k}}_{2}, \overrightarrow{\mathrm{q}}\right)
$$

Turning our attention now to the FHP term, from Eq. (2.21) of I, we find that our usual state-averaging fermion approximation yields precisely the same expression as Eq. (5.2) but with the opposite sign. Hence, in this scheme, the PHB and FHP contributions completely cancel, and the analogy with our treatment of exchange terms in Sec. III leads us to believe that this is probably a very good approximation.

The other term belonging to the complete hole potential (CHP) which contributes in the case of the electron gas, namely the HP term, has already been briefly discussed in Sec. II in connection with the lowdensity limit and in analogy with the comparable Bose gas. We saw there that for low densities the HP term is of major physical significance, whereas for metallic densities it gives only a relatively small effect. However, we include it in our final expressions for the SUB2 equations given below.

We next turn our attention to the PHA and CPP terms given, respectively, by Eqs. (2.24) and (2.22) of I. Again, in our basic fermion approximation, and summing over $\overrightarrow{\mathrm{k}}_{1}$ and $\overrightarrow{\mathrm{k}}_{2}$, these two terms are readily shown to be given by

$$
t_{\mathrm{PHA}} \equiv-2 \sum_{\overrightarrow{\mathrm{k}}_{1} \overrightarrow{\mathrm{k}}_{2} \overrightarrow{\mathrm{k}}} V\left(\overrightarrow{\mathrm{k}}_{2}-\overrightarrow{\mathrm{k}}\right)\left\langle S_{2}^{s_{1} s_{2}}(q)\right\rangle \Theta\left(\overrightarrow{\mathrm{k}}_{1}, \overrightarrow{\mathrm{q}}\right) \Theta\left(-\overrightarrow{\mathrm{k}}_{2}, \overrightarrow{\mathrm{q}}\right) \Theta(-\overrightarrow{\mathrm{k}}, \overrightarrow{\mathrm{q}})
$$

and

$$
t_{\mathrm{CPP}} \equiv-2 \sum_{\overrightarrow{\mathrm{k}}_{1} \overrightarrow{\mathrm{k}}_{2}} \sum_{\overrightarrow{\mathrm{k}}}^{k_{F}} V\left(\overrightarrow{\mathrm{k}}-\overrightarrow{\mathrm{k}}_{2}+\overrightarrow{\mathrm{q}}\right)\left\langle S_{2}^{s_{1} s_{2}}(q)\right\rangle\left[1+2 f_{-\overrightarrow{\mathrm{k}}}\left(\overrightarrow{\mathrm{k}}-\overrightarrow{\mathrm{k}}_{2}+\overrightarrow{\mathrm{q}}\right)\right] \Theta\left(\overrightarrow{\mathrm{k}}_{1}, \overrightarrow{\mathrm{q}}\right) \Theta\left(-\overrightarrow{\mathrm{k}}_{2}, \overrightarrow{\mathrm{q}}\right),
$$


where the function $f$ has been defined in Eq. (3.4). We see that for both small $q$ and for large $q$, the contribution $t_{\mathrm{CPP}}$ is small compared to $t_{\mathrm{PHA}}$. For small values of $q$ this follows from the relation,

$$
\lim _{q \rightarrow 0} f_{\vec{k}}(\vec{q})=-\frac{1}{2}
$$

which is a straightforward consequence of the RPA equations for Coulomb potentials, and for large values of $q$ from comparing the arguments of the potential $V$ in the two expressions. Compared to the PHA term we therefore expect the CPP term to give a small contribution for all momentum transfers. The effect of both terms is to increase the binding energy $|\epsilon|$. Before dealing further with these two terms it proves convenient to turn our attention to the remaining ladder terms in the complete ladder (CLAD) summation for particle-particle and hole-hole interactions within the many-body medium.

The incorporation of the hole-hole ladders (HHLAD) and the mixed ladders (MLAD) is by no means trivial; and preliminary investigations indicate a relatively large repulsive contribution arising from small values of the momentum transfer $q$. However, we have already seen in Sec. IV that in the case of particle-particle ladders (LAD) an appreciable extra effect (i.e., not contained in SUB2 approximation) is obtained by including such ladders also within the RPA terms themselves [i.e., to include diagrams like that of Fig. 8(c)]. These extra effects are contained in the exact equation (2.10) of I for $S_{2}$ by the coupling terms to $S_{3}$ and $S_{4}$, and we have argued that a proper treatment of this interference between the RPA and LAD terms for intermediate momentum transfers is important for good quantitative agreement. However, this interference of RPA and ladder effects is expected to be even stronger for HHLAD (and MLAD) terms than for the particle-particle ladders. The reason for this is quite simply that particle-particle scattering inside the many-body medium must, by the exclusion principle, lead to virtual intermediate states outside the Fermi sea, and hence these LAD terms are important mostly for large-momentum transfers. Conversely, the HHLAD terms describe hole-hole scattering for which the intermediate states are constrained to lie within the Fermi sea, and hence these terms are important for quite small-momentum transfers. We thus see that the HHLAD (and MLAD) terms are of importance precisely in the same low- $q$ regime where RPA terms dominate. By analogy with our treatment in Sec. IV we thus expect it to be of even more importance for these HHLAD and MLAD terms, than it was for the LAD terms, to include the respective higher-order ladder effects from the couplings to $S_{3}$ and $S_{4}$. Unfortunately, these higher terms are not able to be incorporated in a similar fashion to the method used in Sec. IV, but instead one has explicitly to consider three- and four-body equations. Since this is clearly beyond the scope of the present study, we shall assume that the "bare" potential $V$ which appears in the SUB2 terms HHLAD and MLAD is "screened" by these higher coupling effects, such that the large repulsive contribution to the g.s. energy from mainly small values of $q$, is shifted to a much smaller (still repulsive) contribution at intermediate values of $q$. Furthermore, we take the remaining repulsive screened HHLAD and MLAD contribution into account by assuming that it cancels the contribution of the CPP term discussed above. We stress that although these considerations seem to us to be physically well motivated and reasonable, they remain at present largely untested.

The only terms in the SUB2 equations not so far discussed are the extra exchange terms EE1 and EE2 of Eqs. (2.26) and (2.27) of I. Our by now standard approximation scheme immediately leads to an exact cancellation in the parallel-spin contributions from both terms. A partial cancellation of a similar kind also occurs for antiparallel pairs. Since both EE1 and EE2 terms individually are expected to be small for metallic densities, we feel confident in neglecting these terms altogether. We note, however, that in order adequately to include these terms, so that this assertion may be rigorously tested, one must perform a rather complex multidimensional integration.

From our arguments so far, we therefore conclude that our final approximation for the fermion two-particle-two-hole function $S_{2}$ differs from (our third, self-consistent $G$-matrix version of incorporating particle-particle ladders in) Sec. IV only by the inclusion of the PHA and HP terms in addition. In our familiar fermion state-averaging scheme the final equations for $\bar{S}_{2}^{\sigma_{1} \sigma_{2}}$ in this $\mathrm{RPA}^{*}+\mathrm{RPAEX}^{*}+\mathrm{LAD}^{*}+\mathrm{PHA}^{*}+\mathrm{HP}^{*}\left(\left\langle e^{-1}\right\rangle\right)$ scheme, in which the LAD term is incorporated in our self-consistent $G$-matrix version, become

$$
\bar{S}_{2}^{\sigma_{1} \sigma_{2}}(q)=-\frac{1}{\widetilde{e}(q)}\left\{G^{\sigma_{1} \sigma_{2}}(q)+\left[G^{\uparrow \downarrow}(q)-\delta_{\sigma_{1} \sigma_{2}} G^{\uparrow \downarrow}\left(\bar{q}_{\mathrm{ex}}\right)\right]\left[2 \frac{\bar{S}_{2}(q)}{P(q)}+\left(\frac{\bar{S}_{2}(q)}{P(q)}\right)^{2}\right]\right\},
$$


with

$$
\begin{aligned}
\widetilde{e}(q)= & \left\langle e^{-1}\right\rangle^{-1}-\left[\frac{4}{\pi}\right]\left(\alpha r_{s}\right) \int_{0}^{\infty} d q^{\prime} \frac{P\left(q, q^{\prime}\right)}{P(q) P\left(q^{\prime}\right)} \bar{S}_{2}\left(q^{\prime}\right) \\
& -\left[\frac{6}{\pi}\right]\left(\alpha r_{s}\right) \frac{1}{P(q)} \int_{0}^{1} d k_{1} k_{1} \int_{0}^{1} d k_{2} k_{2} \ln \left|\frac{k_{1}+k_{2}}{k_{1}-k_{2}}\right| \hat{\Theta}\left(k_{1}, q\right) \hat{\Theta}\left(k_{2}, q\right) .
\end{aligned}
$$

We remind the reader that the angle-averaged quantity $\hat{\Theta}(k, q)$ was defined in Eqs. (3.6) and (4.12), the "energy denominator" $e$ by Eq. (3.7), and the exchange momentum transfer $\overrightarrow{\mathrm{q}}_{\mathrm{ex}}$ by Eq. (3.8). Furthermore, the state averaging implied in the definition of $\left\langle e^{-1}\right\rangle$ and in the averaged quantity $\bar{q}_{\text {ex }}$ defined by Eq. (4.21) was first defined in Eq. (3.18). The state-averaged quantities $\bar{S}_{2}(q)$, $P(q)$, and $P\left(q, q^{\prime}\right)$ were defined, respectively, in Eqs. (3.15), (3.16), and (4.14). Finally, the stateaveraged $G$-matrix, $G^{\sigma_{1} \sigma_{2}}(q)$ was defined in Eq. (4.16).

The contributions of the HP and PHA terms are included wholly in the second and third terms, respectively, on the right-hand side of Eq. (5.7), and are hence seen in our approximation to "renormalize" the "bare kinetic energy denominator" $e$. We note that the only additional approximation made in including these two new terms was to replace the $\theta$ functions by their angle-averaged values $\hat{\theta}$, just as in the evaluation of the LAD term. We also mention that the RPA ${ }^{*}+\mathrm{HP}^{*}$ approximation of Eq. (2.34), used in Sec. II, is now finally explained.

The final results for the correlation energy, obtained by numerical solution of Eqs. (5.6) and (5.7) in the metallic-density regime, are shown in Table III, and the corresponding state-averaged $\bar{S}_{2}$ functions are shown for both parallel- and antiparallelspin pairs in Fig. 11 at a value $r_{s}=4$. By way of comparison we also show in Table III some results obtained by Ceperley and Alder, ${ }^{25}$ using the Green's-function Monte Carlo (GFMC) method which is believed to give essentially exact results for the lowest eigenvalue of the many-body Schrödinger equation. The agreement with our results is both striking and very gratifying, but the obvious question presents itself as to whether our relatively simple calculation (which "only" involves solving two nonlinear one-dimensional integral equations) can really be expected to give the exact eigenvalue of the many-body Schrödinger equation, in this density regime, with an accuracy of about $1 \%$. In order to attempt to answer this question we must address ourselves to three separate issues, viz., (i) the adequacy and accuracy of the fermion state-averaging approximation, (ii) the adequacy of omitting and adding terms to the SUB2 approximation, and (iii) the adequacy of the SUB2 approximation itself.

Concerning point (i) we may review again the evidence in favor of our fermion averaging procedure being very accurate indeed. Thus we point out again the very striking agreement shown in Fig. 6, where the crosses, which denote exact numerical solutions in a particular approximation [RPA + RPAEX(1)], are seen to lie almost exactly on the corresponding curve which represents the results of our fermion averaging procedure in this same case. In Sec. III it became clear that this result, for the RPA + RPAEX approximation, is valid for metallic densities. The corresponding error for the correlation energy may be seen from columns 6 and 7 of Table I to be precisely of the same order as the error we found in Table III for our final results in comparison with the GFMC re-

\begin{tabular}{|c|c|c|c|c|c|}
\hline Calculation & 1 & 2 & $\begin{array}{l}r_{s} \\
3\end{array}$ & 4 & 5 \\
\hline $\begin{array}{c}\mathrm{CC}^{\mathrm{a}} \\
\mathrm{GFMC}^{\mathrm{b}}\end{array}$ & $\begin{array}{l}-0.123 \\
-0.121\end{array}$ & $\begin{array}{l}-0.0917 \\
-0.0902\end{array}$ & -0.0751 & -0.0644 & $\begin{array}{l}-0.0568 \\
-0.0563\end{array}$ \\
\hline
\end{tabular}

TABLE III. Final results for the Fermi correlation energy.

${ }^{a}$ From the present coupled-cluster calculation using Eqs. (5.6) and (5.7).

${ }^{b}$ From the Green's-function Monte Carlo calculation of Ceperley and Alder (Ref. 25). 


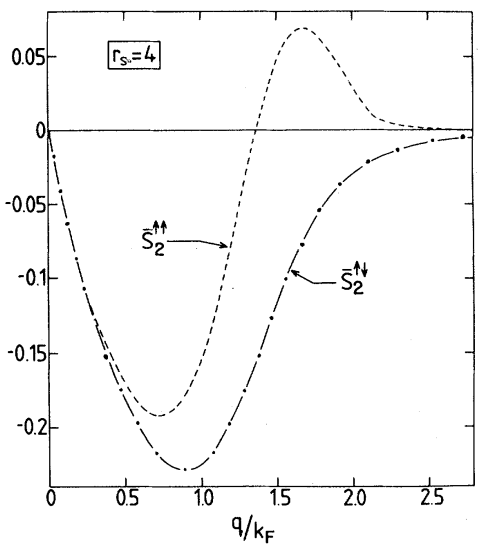

FIG. 11. As in Fig. 7, but in the RPA* + RPAEX* $+\mathrm{LAD}^{*}+\mathrm{PHA}^{*}+\mathrm{HP}^{*}\left(\left\langle e^{-1}\right\rangle\right)$ approximation of Eqs. (5.6) and (5.7), using the self-consistent $G$-matrix version of incorporating ladders.

sults. However, the approximation in the RPA + RPAEX case has been put to a much more severe test in Fig. 6 as far as point (i) is concerned, since we test here the adequacy of the averaging scheme for the entire momentum distribution and not just for its integrated value, the correlation energy. We have argued in Secs. IV and V that the fermion averaging approximation is expected to be comparably accurate for the additional terms considered; but it must be admitted we have no detailed checks here, and it could easily happen that a piling up of various small errors could lead to a final error appreciably larger than $1 \%$.

Concerning point (ii) above we would only wish to emphasize that the agreement observed in Table III was certainly not achieved by a process of omitting and adding terms until the most favorable result was obtained. In fact, our final results reported in Table III were obtained (as final results) before we learned about the GFMC results. The only other point that we wish to reiterate concerning the adequacy of omitting and adding terms to the SUB2 equations, is that the omission of the hole-hole and mixed particle-particle and hole-hole ladder (HHLAD and MLAD) terms is without doubt the prime candidate for the largest remaining source of error.

Finally, concerning point (iii) on the adequacy of the SUB2 approximation itself, we remind the reader of our previous discussion in Sec. II of I. We also recall that certain aspects of the coupling to the three- and four-body clusters described by $S_{3}$ and $S_{4}$, and which goes beyond SUB2, have also been incorporated in our final results. Thus in Sec. IV we made explicit use of this coupling in our third and final self-consistent $G$-matrix version of incorporating particle-particle ladder effects, and in Sec. $V$ we made implicit use of the coupling in our discussion of the effects of hole-hole and mixed ladder terms. That other effects of the coupling to $S_{3}$ and $S_{4}$ are unimportant in the metallicdensity regime, must also strictly speaking be listed as a final assumption.

It is clear that too many sources of error are involved for us to answer more quantitatively the question posed initially. We believe that although it is possible we may have been fortunate in obtaining such accurate final results, the actual physics of the electron gas in the metallic-density regime is nevertheless very well represented in our calculation, in that we believe that no important effect has been neglected. A detailed comparison of Figs. 9-11 shows the corresponding curves to be asymptotically the same for large $q$. This clearly means that for large $q$, on the one hand, the functions $\bar{S}_{2}$ are determined by ladders and exchange effects only. The influence of the RPA terms (which themselves dominate at small $q$, on the other hand) is in fact seen to become unimportant somewhere in the range $1.5 \lesssim q \lesssim 2.0$, as indeed is also seen from Fig. 6 of I. It is precisely this feature which enables Lowy and Brown ${ }^{21}$ to interpolate quite successfully between the short- and long-range limits. Our whole treatment aimed to treat these basic features with some care, and we dealt separately with RPA and exchange effects in Sec. III and with ladder effects in Sec. IV. Our emphasis and motivation here has been first of all to treat the interference between these effects, in the intermediate-range region, as accurately as possible. A measure of this interference was first seen in Fig. 6 as the difference between the curves labelled RPA* + RPAEX ${ }^{*}$ and RPA* + $\operatorname{RPAEX}^{*}(1)$, and in Sec. IV again the considerations of interference between the short- and longrange phenomena were instrumental in forcing us to go beyond SUB2 and to include the relevant contributions from the coupling to higher clusters. We finally note that the eventual remarkable success of our treatment must properly be placed in the context of the previous discussion.

\section{CONCLUSIONS}

Just as we saw in I that the coupled-cluster formalism could most successfully be applied to the high-density one-component electron plasma, we have now seen in the present work that the formalism works equally well for both the charged Bose 
plasma and for the electron plasma at low and intermediate densities. In particular for metallic densities we obtained the striking result of about $1 \%$ agreement for the correlation energy with recent, essentially exact, calculations of the GFMC type. Since we do not wish to repeat the discussion of these results already given at the end of Sec. V, we limit ourselves now to a brief examination of Secs. II-IV.

Based on an analogy with boson systems, results were presented in Sec. II for the low-density electron plasma for a particularly simple approximation which included only RPA and hole-potential (HP) effects. We saw explicitly that our intrinsically fluidlike and everywhere translationallyinvariant approach could nevertheless provide a good (at least qualitative) description for both bosons and fermions of the low-density solid phase, as well as for the high-density gaseous phase. We believe that the coupled-cluster formalism has hence provided us for the first time with a unified description of the entire density range at zero temperature. It is interesting to point out that historically the HP term was essentially included for the first time by Bethe, Brandow, and Petschek ${ }^{26}$ in the context of a perturbation-theoretic treatment of the nuclear matter problem. In that context the inclusion of the HP term may be thought of as incorporating the effect of the phenomenological single-particle nuclear shell-model potential in a self-consistent microscopic calculation based on the two-body potential as input. Similarly, in the present context we may view the HP term as taking into account an average self-consistent onebody potential, in which the electrons may solidify as the kinetic energy vanishes. In nuclear matter calculations the incorporation of HP effects has often been criticized on the grounds that a similar average potential for particle states (outside the Fermi sea) is much harder to calculate and for that reason usually neglected. Indeed in the present case also, the consistent inclusion of such an additional comparable particle potential term would certainly entail the solution of at least a three-body equation and hence certainly take us outside the SUB2 scheme. [As a lengthy aside we note that by the comparable particle potential here we do not mean the factorizable SUB2 particle-potential insertions of Eq. (2.22) and diagrams 1(j) -1(1) of Fig. 1 of I; but rather the one obtained from the factorizable hole-potential insertions given by Eq. (2.21) and diagrams $1(\mathrm{~g})-1(\mathrm{i})$ of Fig. 1 of $\mathrm{I}$, by "time reversing" the external hole lines into parti- cle lines. We note also that there is no real lack of symmetry between particles and holes here, since just as the comparable particle potential to the factorizable SUB2 hole potential is itself a nonfactorizable potential that arises only when couplings to higher subsystems are considered, so the same is true when the roles of particles and holes are reversed.] To return, this is in fact the main reason why for the low-density fermion case we did not try to develop a quantitatively better approximation than the very simple one considered in Sec. II. It is clear on all counts that at low densities the coupling of $S_{2}$ to $S_{3}$ and $S_{4}$ may not be neglected, but what we wish to stress most is that the simple RPA + HP approximation already contains the essential physics of this region, and gives the correct qualitative and analytic behavior expected of a solid.

The state-averaging approximation developed for the case of Fermi statistics in Sec. III has also proved to be very successful. In essence it reduces the complexity of the fermion equations to that of the corresponding boson system. We estimate that in the range of metallic densities where its accuracy seems to be of the order of $1 \%$, it allows a reduction in computing time of a factor of at least 100. Furthermore, it even becomes asymptotically exact in the most interesting physical limit, namely as $q$ approaches zero.

In Sec. IV one feature of central importance which was stressed was the interference at intermediate separations of the long-range RPA screening effect with the effects of the short-range particle-particle ladders. It was this feature which first forced us to go beyond the SUB2 approximation and to take into account some important effects of the coupling of $S_{2}$ to $S_{3}$ and $S_{4}$. We adopted the viewpoint that within our SUB2 approximation scheme, $S_{3}$ and $S_{4}$ may be regarded as essentially free functions, and we determined them by a comparison of our three- and four-body subsystem wave functions with a wave function of the Jastrow cluster type. This latter cluster wave function was assumed to be of the correct form in certain asymptotic cases, namely for large distances between various particles in the subsystem under consideration. Although this procedure seems to be entirely legitimate, it is nevertheless not entirely satisfactory since it ought to be possible to make the needed connection wholly within the coupledcluster formalism. Attempts to prove the necessary relations about various asymptotic properties of the subsystem amplitudes $S_{n}$ as exact statements 
have so far only been partially successful. For the interested reader we add only that the required theorems are related to a well-known theorem concerning the asymptotic behavior of the liquidstructure function, which is itself difficult to prove formally. We hope to be able to report elsewhere on this matter at a later date.

In conclusion we add only that we have reported here on a microscopic calculation of the onecomponent electron plasma which seems to our minds to be more ambitious than any previous calculation in that it incorporates more (and indeed if pressed we would go further and say all) of the relevant physics of the problem. At the same time it is clearly also more successful than any competing calculation, insofar as the high-density limit is exact; the agreement in the metallic-density regime with the essentially exact GFMC results is second to none, and the low-density limit is at least analytically that of a solid and not a fluid. All in all, we could hardly wish for a more satisfactory outcome. It would seem likely that a careful application of the method employed here to fermion systems other than the one-component plasma might also offer equally promising results.

\section{ACKNOWLEDGMENTS}

This research was supported in part by the Deutsche Forschungsgemeinschaft and by the Science Research Council of Great Britain under Grant No. GR/B/5778.1. The bulk of the work presented here was performed while both authors were at the Nuclear Science Division, Lawrence Berkeley Laboratory, University of California, Berkeley, California 94720.

\section{APPENDIX A: EVALUATION OF $\epsilon_{c}^{\mathrm{RPAEX}(1)}$}

From Eq. (3.12) we have

$$
\epsilon_{c}^{\mathrm{RPAEX}(1)}=\frac{3}{16 \pi^{5}} \int \frac{d \overrightarrow{\mathrm{q}}}{q^{2}} \int_{\widetilde{\Gamma}} d \vec{\kappa}_{1} \int_{\widetilde{\Gamma}} d \vec{\kappa}_{2} \frac{\widetilde{g}_{R}\left(\vec{\kappa}_{1}\right) \widetilde{g}_{R}\left(\vec{\kappa}_{2}\right)}{\left|\vec{\kappa}_{1}+\vec{\kappa}_{2}\right|^{2}\left(\overrightarrow{\mathrm{q}} \cdot \vec{\kappa}_{1}+\overrightarrow{\mathrm{q}} \cdot \vec{\kappa}_{2}\right)},
$$

where

$$
\vec{\kappa} \in \widetilde{\Gamma} \Longrightarrow\left\{\begin{array}{l}
\left|\vec{\kappa}-\frac{1}{2} \vec{q}\right|<1 \\
\left|\vec{\kappa}+\frac{1}{2} \vec{q}\right|>1 .
\end{array}\right.
$$

It is now useful to specify the vectors $\vec{\kappa}_{1}$ and $\vec{\kappa}_{2}$ in a cylindrical polar coordinate system with axis along the direction of $\vec{q}$ and origin at the center of the two Fermi spheres implied by the integration region $\widetilde{\Gamma}$. Writing thus $\vec{\kappa}_{i}=\left(x_{i}, \rho_{i}, \theta_{i}\right)$ with $i=1,2$ and $\kappa_{i x}=\rho_{i} \sin \theta_{i}, \kappa_{i y}=\rho_{i} \cos \theta_{i}, \kappa_{i z}=x_{i}$, we may rewrite Eq. (A2) as

$$
\begin{aligned}
\epsilon_{c}^{\mathrm{RPAEX}(1)}=\frac{3}{4 \pi^{4}} \int_{0}^{\infty} \frac{d q}{q} \int_{\widetilde{\Gamma}} d \vec{\kappa}_{1} \widetilde{g}_{R}\left(\vec{\kappa}_{1}\right) \int_{\widetilde{\Gamma}} d \vec{\kappa}_{2} \widetilde{g}_{R}\left(\vec{\kappa}_{2}\right)\left(x_{1}+x_{2}\right)^{-1} \\
\times\left[\left(x_{1}+x_{2}\right)^{2}+\rho_{1}^{2}+\rho_{2}^{2}+2 \rho_{1} \rho_{2} \cos \left(\theta_{1}-\theta_{2}\right)\right]^{-1} .
\end{aligned}
$$

It is straightforward to show that the integrations over the region $\widetilde{\Gamma}$ can be expressed as

$$
\int_{\widetilde{\Gamma}} d \vec{\kappa}_{1} \rightarrow \int_{0}^{2 \pi} d \theta_{i} \int_{Q_{-}}^{Q_{+}} d x_{i} \int_{0}^{\rho_{i}^{+}} \rho_{i} d \rho_{i}+\Theta(2-q) \int_{0}^{2 \pi} d \theta_{i} \int_{0}^{Q_{-}} d x_{i} \int_{\rho_{i}^{-}}^{\rho_{i}^{+}} \rho_{i} d \rho_{i}
$$

where

$$
\rho_{i}^{+} \equiv\left[1-\left(\frac{1}{2} q \mp x_{i}\right)^{2}\right]^{1 / 2}
$$

and

$$
Q_{ \pm} \equiv\left|\frac{1}{2} q \pm 1\right| \text {. }
$$

With the use of Eqs. (A4) and (A5) the two angular integrals in Eq. (A3) can now trivially be performed, 
since from I the function $\widetilde{g}_{R}(\vec{\kappa})=\widetilde{g}_{R}(x ; q)$ does not depend on the coordinates $\rho$ or $\theta$. Making the trivial substitution $\rho_{i}^{2} \equiv y_{i}$ leads to the final results

$$
\epsilon_{c}^{\mathrm{RPAEX}(1)}=\frac{3}{4 \pi^{2}} \int_{0}^{\infty} d q Z(q),
$$

where

$$
Z(q)=\frac{1}{q} \int_{Q_{-}}^{Q_{+}} d x_{1} \int_{Q_{-}}^{Q_{+}} d x_{2} \int_{0}^{y_{1}^{+}} d y_{1} \int_{0}^{y_{2}^{+}} d y_{2} \frac{\widetilde{g}_{R}\left(x_{1} ; q\right) \widetilde{g}_{R}\left(x_{2} ; q\right)}{x_{1}+x_{2}} \frac{1}{F^{1 / 2}}, \quad q \geq 2
$$

and

$$
\begin{aligned}
Z(q)= & \frac{1}{q}\left(\int_{0}^{Q_{-}} d x_{1} \int_{y_{1}^{-}}^{y_{1}^{+}} d y_{1}+\int_{Q_{-}}^{Q_{+}} d x_{1} \int_{0}^{y_{1}^{+}} d y_{1}\right)\left(\int_{0}^{Q_{-}} d x_{2} \int_{y_{2}^{-}}^{y_{2}^{+}} d y_{2}+\int_{Q_{-}}^{Q_{+}} d x_{2} \int_{0}^{y_{2}^{+}} d y_{2}\right) \\
& \times \frac{\widetilde{g}_{R}\left(x_{1} ; q\right) \widetilde{g}_{R}\left(x_{2} ; q\right)}{x_{1}+x_{2}} \frac{1}{F^{1 / 2}}, \quad q<2
\end{aligned}
$$

where

$$
y_{i}^{ \pm} \equiv\left(\rho_{i}^{ \pm}\right)^{2}
$$

and

$$
F \equiv\left(x_{1}+x_{2}\right)^{4}+2\left(x_{1}+x_{2}\right)^{2}\left(y_{1}+y_{2}\right)+\left(y_{1}-y_{2}\right)^{2} .
$$

From Eqs. (3.56e), (3.57), and (3.19) of I, we also have

$$
\widetilde{g}_{R}(x ; q)=\exp \left[-\frac{x}{\pi} \int_{0}^{\infty} d t \frac{\ln \widetilde{E}(t ; q)}{t^{2}+x^{2}}\right),
$$

where

$$
\widetilde{E}(t ; q)=1+\frac{2 \alpha r_{s}}{\pi q^{2}} G(t ; q)
$$

and

$$
G(t ; q)=1+\frac{1}{2 q}\left(1-\frac{1}{4} q^{2}+t^{2}\right) \ln \left(\frac{\left(\frac{1}{2} q+1\right)^{2}+t^{2}}{\left(\frac{1}{2} q-1\right)^{2}+t^{2}}\right)+t\left[\tan ^{-1}\left(\frac{\frac{1}{2} q-1}{t}\right)-\tan ^{-1}\left(\frac{\frac{1}{2} q+1}{t}\right)\right] .
$$

Further evaluation of Eqs. (A7) - (A13) is performed by numerical integration.

\section{APPENDIX B: EVALUATION OF $\langle e\rangle$ and $\left\langle e^{-1}\right\rangle$}

Using Eqs. (3.7), (3.16), and (3.18) and proceeding to the usual thermodynamic limit wherein sums over momentum states may be replaced by corresponding (dimensionless) integrals,

$$
\sum_{\overrightarrow{\mathrm{k}}} \rightarrow \frac{\Omega k_{F}^{3}}{(2 \pi)^{3}} \int d \overrightarrow{\mathrm{k}}=\frac{3 N}{8 \pi} \int d \overrightarrow{\mathrm{k}}
$$

we have

$$
\langle e\rangle=\left(\frac{4 \pi}{3} P(q)\right]^{-2} \int d \overrightarrow{\mathrm{k}}_{1} \int d \overrightarrow{\mathrm{k}}_{2} \overrightarrow{\mathrm{q}} \cdot\left(\overrightarrow{\mathrm{k}}_{1}-\overrightarrow{\mathrm{k}}_{2}+\overrightarrow{\mathrm{q}}\right) \Theta\left(\overrightarrow{\mathrm{k}}_{1}, \overrightarrow{\mathrm{q}}\right) \Theta\left(-\overrightarrow{\mathrm{k}}_{2}, \overrightarrow{\mathrm{q}}\right) .
$$

By writing $e$ in the form 


$$
e=\frac{1}{2}\left[\left(\overrightarrow{\mathrm{k}}_{1}+\overrightarrow{\mathrm{q}}\right)^{2}-k_{1}^{2}+\left(\overrightarrow{\mathrm{k}}_{2}-\overrightarrow{\mathrm{q}}\right)^{2}-k_{2}^{2}\right],
$$

the integral in Eq. (B2) can be written as the sum of two terms in each of which the integrations on $\overrightarrow{\mathrm{k}}_{1}$ and $\overrightarrow{\mathrm{k}}_{2}$ are decoupled. Furthermore, by the substitution $\overrightarrow{\mathrm{k}}_{1} \rightleftarrows-\overrightarrow{\mathrm{k}}_{2}$ in the second term, both terms are seen to be identical, and hence we find

$$
\langle e\rangle=\left(\frac{4 \pi}{3} P(q)\right]^{-2} \int d \overrightarrow{\mathrm{k}}_{1}\left[\left(\overrightarrow{\mathrm{k}}_{1}+\overrightarrow{\mathrm{q}}\right)^{2}-k_{1}^{2}\right] \Theta\left(1-k_{1}\right) \Theta\left(\left|\overrightarrow{\mathrm{k}}_{1}+\overrightarrow{\mathrm{q}}\right|-1\right) \int d \overrightarrow{\mathrm{k}}_{2} \Theta\left(-\overrightarrow{\mathrm{k}}_{2}, \overrightarrow{\mathrm{q}}\right) .
$$

In Eq. (B4) the integral on $\overrightarrow{\mathrm{k}}_{2}$ can be immediately performed using Eqs. (B1) and (3.16). The integral on $\overrightarrow{\mathrm{k}}_{1}$ in Eq. (B4) is most easily performed by writing the product of $\Theta$ functions as

$$
\Theta\left(1-k_{1}\right) \Theta\left(\left|\vec{k}_{1}+\vec{q}\right|-1\right)=\Theta\left(1-k_{1}\right)-\Theta\left(1-k_{1}\right) \Theta\left(1-\left|\vec{k}_{1}+\vec{q}\right|\right) \text {. }
$$

Under the substitution $\overrightarrow{\mathrm{k}}_{1} \rightarrow \overrightarrow{\mathrm{k}}_{1}^{\prime}=-\overrightarrow{\mathrm{k}}_{1}-\overrightarrow{\mathrm{q}}$ it is clear that the second term on the right-hand side of Eq. (B5) is even, whereas the remainder of the integrand in the $\overrightarrow{\mathrm{k}}_{1}$ integral in Eq. (B4) is odd. Hence we find,

$$
\langle e\rangle=\left[\frac{4 \pi}{3} P(q)\right]^{-1} \int d \overrightarrow{\mathrm{k}}_{1}\left(q^{2}+2 \overrightarrow{\mathrm{k}}_{1} \cdot \overrightarrow{\mathrm{q}}\right) \Theta\left(1-k_{1}\right)
$$

and trivial evaluation leads to the final result

$$
\langle e\rangle=q^{2} / P(q) \text {. }
$$

Again from Eqs. (3.7), (3.16), and (3.18) we have

$$
\left\langle e^{-1}\right\rangle=\left(\frac{4 \pi}{3} P(q)\right]^{-2} \int d \overrightarrow{\mathrm{k}}_{1} \int d \overrightarrow{\mathrm{k}}_{2} \frac{1}{\overrightarrow{\mathrm{q}} \cdot\left(\overrightarrow{\mathrm{k}}_{1}-\overrightarrow{\mathrm{k}}_{2}+\overrightarrow{\mathrm{q}}\right)} \Theta\left(\overrightarrow{\mathrm{k}}_{1}, \overrightarrow{\mathrm{q}}\right) \Theta\left(-\overrightarrow{\mathrm{k}}_{2}, \overrightarrow{\mathrm{q}}\right) .
$$

Making the simple change of variables,

$$
\overrightarrow{\mathrm{k}}_{1}=\vec{\kappa}_{1}-\frac{1}{2} \overrightarrow{\mathrm{q}}, \quad \overrightarrow{\mathrm{k}}_{2}=-\vec{\kappa}_{2}+\frac{1}{2} \overrightarrow{\mathrm{q}}
$$

enables us to rewrite Eq. (B7) as

$$
\left\langle e^{-1}\right\rangle=\left[\frac{4 \pi}{3} P(q)\right]^{-2} \int_{\widetilde{\Gamma}} d \vec{\kappa}_{1} \int_{\widetilde{\Gamma}} d \vec{\kappa}_{2} \frac{1}{\overrightarrow{\mathrm{q}} \cdot\left(\vec{\kappa}_{1}+\vec{\kappa}_{2}\right)},
$$

where $\widetilde{\Gamma}$ is the integration region indicated in Eq. (A2). Choosing the same cylindrical polar coordinate system as in Appendix A, and using Eq. (A4), readily enables us to rewrite Eq. (B9) as

$$
\left\langle e^{-1}\right\rangle=\left(\frac{4 \pi}{3} P(q)\right]^{-2} \int_{\widetilde{L}} 2 \pi q \widetilde{N}\left(x_{1}\right) d x_{1} \int_{\tilde{L}} 2 \pi q \tilde{N}\left(x_{2}\right) d x_{2} \frac{1}{q\left(x_{1}+x_{2}\right)},
$$

where the integration region $\widetilde{L}$ is specified by

$$
x \in \widetilde{L} \Longrightarrow\left\{\begin{array}{l}
0 \leq x \leq \frac{1}{2} q+1, \quad q<2 \\
\frac{1}{2} q-1 \leq x \leq \frac{1}{2} q+1, \quad q \geq 2
\end{array}\right.
$$

and in this region, the function $\widetilde{N}(x)$ is given by

$$
\widetilde{N}(x)= \begin{cases}x, \quad 0 \leq x \leq 1-\frac{1}{2} q, & q<2 \\ {\left[1-\left(\frac{1}{2} q-x\right)^{2}\right] / 2 q,} & 1-\frac{1}{2} q \leq x \leq 1+\frac{1}{2} q, \quad q<2 \\ {\left[1-\left(\frac{1}{2} q-x\right)^{2}\right] / 2 q,} & \frac{1}{2} q-1 \leq x \leq \frac{1}{2} q+1, \quad q \geq 2 .\end{cases}
$$


The integrals in Eq. (B10) can be further simplified by using the trivial identity,

$$
\frac{2 \pi}{x_{1}+x_{2}}=\int_{0}^{\infty} d y \frac{2 x_{1}}{\left(x_{1}^{2}+y^{2}\right)} \frac{2 x_{2}}{\left(x_{2}^{2}+y^{2}\right)},
$$

to rewrite Eq. (B10) as

$$
\left\langle e^{-1}\right\rangle=\left(\frac{4 \pi}{3} P(q)\right]^{-2} 2 \pi q \int_{0}^{\infty} d y G^{2}(y ; q),
$$

where

$$
G(y ; q)=\int_{\widetilde{L}} d x \widetilde{N}(x) \frac{2 x}{x^{2}+y^{2}} .
$$

Direct evaluation of Eq. (B15), using Eqs. (B11) and (B12) gives the explicit result for the function $G(y ; q)$ quoted already in Eq. (A13), and which is valid for all values of $q$. Equation (B14) is not susceptible to further analytic reduction, but is readily evaluated numerically.

\section{APPENDIX C: EVALUATION OF $\left\langle q_{\mathrm{ex}}^{-2} e^{-1}\right\rangle$}

In Sec. III where we considered the inclusion of exchange effects we found it necessary to evaluate the average $\left\langle q_{\mathrm{ex}}^{-2} e^{-1}\right\rangle$ of the expression

$$
q_{\mathrm{ex}}^{-2} e^{-1} \equiv\left\{\left(\overrightarrow{\mathrm{k}}_{1}-\overrightarrow{\mathrm{k}}_{2}+\overrightarrow{\mathrm{q}}\right)^{2}\left[\overrightarrow{\mathrm{q}} \cdot\left(\overrightarrow{\mathrm{k}}_{1}-\overrightarrow{\mathrm{k}}_{2}+\overrightarrow{\mathrm{q}}\right)\right]\right\}^{-1},
$$

where the average is to be taken according to definition (3.18). Passing to the thermodynamic limit by use of Eq. (B1) and making the same change of variables as in Eq. (B8) readily yields the expression,

$$
\left\langle q_{\mathrm{ex}}^{-2} e^{-1}\right\rangle=\left[\frac{4 \pi}{3} P(q)\right]^{-2} \int_{\widetilde{\Gamma}} d \vec{\kappa}_{1} \int_{\widetilde{\Gamma}} d \vec{\kappa}_{2} \frac{1}{\left(\vec{\kappa}_{1}+\vec{\kappa}_{2}\right)^{2}} \frac{1}{\left(\overrightarrow{\mathrm{q}} \cdot \vec{\kappa}_{1}+\overrightarrow{\mathrm{q}} \cdot \vec{\kappa}_{2}\right)},
$$

where the integration region $\widetilde{\Gamma}$ has been defined in Eq. (A2). Comparing Eq. (C2) with Eq. (A1) enables us to use the same procedure for the evaluation of Eq. (C2) as already developed in Appendix A. We quote only the final result,

$$
\left\langle q_{\mathrm{ex}}^{-2} e^{-1}\right\rangle=\left[\frac{4}{3} P(q)\right]^{-2} \frac{1}{q} \int_{Q_{-}}^{Q_{+}} d x_{1} \int_{Q_{-}}^{Q_{+}} d x_{2} \int_{0}^{y_{1}^{+}} d y_{1} \int_{0}^{y_{2}^{+}} d y_{2} \frac{F^{-1 / 2}}{\left(x_{1}+x_{2}\right)}, q>2
$$

and

$$
\begin{aligned}
\left\langle q_{\mathrm{ex}}^{-2} e^{-1}\right\rangle= & {\left[\frac{4}{3} P(q)\right]^{-2} \frac{1}{q}\left[\int_{0}^{Q_{-}} d x_{1} \int_{y_{1}^{-}}^{y_{1}^{+}} d y_{1}+\int_{Q_{-}}^{Q_{+}} d x_{1} \int_{0}^{y_{1}^{+}} d y_{1}\right) } \\
& \times\left(\int_{0}^{Q_{-}} d x_{2} \int_{y_{2}^{-}}^{y_{2}^{+}} d y_{2}+\int_{Q_{-}}^{Q_{+}} d x_{2} \int_{0}^{y_{2}^{+}} d y_{2}\right) \frac{F^{-1 / 2}}{\left(x_{1}+x_{2}\right)}, \quad q \geq 2
\end{aligned}
$$

where $Q_{ \pm}, y_{i}^{ \pm}$, and $F$ are defined in Eqs. (A6), (A9), and (A10). The further evaluation of Eqs. (C3) is performed numerically.

${ }^{1}$ R. F. Bishop and K. H. Lührmann, Phys. Rev. B $\underline{17}$, 3757 (1978).

${ }^{2}$ R. F. Bishop and K. H. Lührmann (unpublished).

${ }^{3}$ K. H. Lührmann, Ann. Phys. (N.Y.) 103, 253 (1977).

${ }^{4}$ H. Kümmel, K. H. Lührmann, and J. G. Zabolitzky, Phys. Rep. 36C, 1 (1978).

${ }^{5}$ L. L. Foldy, Phys. Rev. 124, 649 (1961); 125, 2208
(1962).

${ }^{6}$ K. A. Brueckner, Phys. Rev. 156, 204 (1967).

${ }^{7}$ E. P. Wigner, Phys. Rev. 46, 1002 (1934).

${ }^{8} \mathrm{D}$. Pines, The Many-Body Problem (Benjamin, New York, 1962), p. 43.

${ }^{9}$ W. J. Carr, Jr., R. A. Coldwell-Horsfall, and A. E. Fein, Phys. Rev. 124, 747 (1961). 
${ }^{10}$ E. Feenberg, J. Low Temp. Phys. 16, 125 (1974).

${ }^{11}$ M. Gell-Mann and K. A. Brueckner, Phys. Rev. 106, 364 (1957).

12J. G. Zabolitzky, Phys. Rev. B 22, 2353 (1980).

${ }^{13}$ D. L. Freeman, Phys. Rev. B 15, 5512 (1977).

${ }^{14}$ L. Onsager, L. Mittag, and M. J. Stephen, Ann. Phys. (Leipzig) 18, 71 (1966).

15J. Hubbard, Proc. R. Soc. London Ser. A $\underline{243}, 336$ (1957).

${ }^{16}$ K. S. Singwi, M. P. Tosi, R. H. Land, and A. Sjölander, Phys. Rev. 176, 589 (1968); K. S. Singwi and M. P. Tosi, ibid. 181, 784 (1969); K. S. Singwi, A. Sjölander, M. P. Tosi, and R. H. Land, Solid State Commun. 7, 1503 (1969); R. Lobo, K. S. Singwi, and M. P. Tosi, Phys. Rev. 186, 470 (1969); and K. S. Singwi, A. Sjölander, M. P. Tosi, and R. H. Land, Phys. Rev. B 1 , 1044 (1970).

17J. B. Pendry, J. Phys. C $\underline{3}, 1711$ (1970); T. P. Fishlock and J. B. Pendry, ibid. $\underline{6}, 1909$ (1973).

${ }^{18} \mathrm{H}$. Yasuhara, Solid State Commun. 11, 1481 (1972).

${ }^{19}$ B. B. J. Hede and J. P. Carbotte, Can. J. Phys. $\underline{50}$, 1756 (1972).

20P. Vashishta and K. S. Singwi, Phys. Rev. B $\underline{6}, 875$ (1972).

${ }^{21}$ D. N. Lowy and G. E. Brown, Phys. Rev. B $\underline{12}, 2138$ (1975).

${ }^{22}$ K. Bedell and G. E. Brown, Phys. Rev. B 17,4512 (1978).

${ }^{23}$ B. Day, Rev. Mod. Phys. 39, 719 (1967).

${ }^{24}$ K. H. Lührmann and H. Kümmel, Nucl. Phys. A194, 225 (1972).

${ }^{25}$ D. M. Ceperley and B. J. Alder, Lawrence Berkeley Laboratory Report No. LBL-10813 (UC-4) (unpublished).

${ }^{26}$ H. A. Bethe, B. H. Brandow, and A. G. Petschek, Phys. Rev. 129, 225 (1963). 